\title{
ATR maintains chromosomal integrity during postnatal cerebellar neurogenesis and is required for medulloblastoma formation
}

\author{
Patrick Y. Lang ${ }^{1,2}$, Gouri J. Nanjangud ${ }^{3}$, Marina Sokolsky-Papkov ${ }^{4}$, Christine Shaw ${ }^{3}$, Duhyeong Hwang ${ }^{4}$, \\ Joel S. Parker ${ }^{5,6}$, Alexander V. Kabanov ${ }^{4}$ and Timothy R. Gershon ${ }^{2,6,7, *}$
}

\begin{abstract}
Microcephaly and medulloblastoma may both result from mutations that compromise genomic stability. We report that ATR, which is mutated in the microcephalic disorder Seckel syndrome, sustains cerebellar growth by maintaining chromosomal integrity during postnatal neurogenesis. Atr deletion in cerebellar granule neuron progenitors (CGNPs) induced proliferation-associated DNA damage, p53 activation, apoptosis and cerebellar hypoplasia in mice. Co-deletions of either p53 or Bax and Bak prevented apoptosis in Atr-deleted CGNPs, but failed to fully rescue cerebellar growth. ATR-deficient CGNPs had impaired cell cycle checkpoint function and continued to proliferate, accumulating chromosomal abnormalities. RNA-Seq demonstrated that the transcriptional response to ATR-deficient proliferation was highly p53 dependent and markedly attenuated by p53 co-deletion. Acute ATR inhibition in vivo by nanoparticle-formulated VE-822 reproduced the developmental disruptions seen with Atr deletion. Genetic deletion of Atr blocked tumorigenesis in medulloblastomaprone SmoM2 mice. Our data show that p53-driven apoptosis and cell cycle arrest - and, in the absence of p53, non-apoptotic cell death - redundantly limit growth in ATR-deficient progenitors. These mechanisms may be exploited for treatment of CGNP-derived medulloblastoma using ATR inhibition.
\end{abstract}

KEY WORDS: Microcephaly, Medulloblastoma, Chromosomal instability, ATR, Neural progenitor, DNA damage, Mouse

\section{INTRODUCTION}

Appropriate brain development requires genomic fidelity. Diverse mutations that increase DNA damage can restrict brain growth, causing microcephaly (Lee and McKinnon, 2007; McMahon et al., 2014; Orii et al., 2006; Rosin et al., 2015; Williams et al., 2015). Combined with $p 53$ deletion, mutations in DNA repair genes may produce unrestricted growth in medulloblastoma (Frappart et al., 2009; Lee and McKinnon, 2002; Tong et al., 2003), a malignant tumor of neural progenitors. The serine/threonine kinase ATR

\footnotetext{
${ }^{1}$ Department of Cell Biology and Physiology, School of Medicine, University of North Carolina, Chapel Hill, NC 27599, USA. ${ }^{2}$ Department of Neurology, UNC School of Medicine, University of North Carolina, Chapel Hill, NC 27599, USA. ${ }^{3}$ Molecular Cytogenetics Core Facility, Memorial Sloan Kettering Research Center, New York, NY 10021, USA. ${ }^{4}$ Center for Nanotechnology in Drug Delivery and Division of Molecular Pharmaceutics, Eshelman School of Pharmacy, University of North Carolina, Chapel Hill, NC 27599, USA. ${ }^{5}$ Department of Genetics, School of Medicine, University of North Carolina, Chapel Hill, NC 27599, USA. ${ }^{6}$ Lineberger Comprehensive Cancer Center, University of North Carolina, Chapel Hill, NC 27599, USA. ${ }^{7}$ Neuroscience Center, University of North Carolina, Chapel Hill, NC 27599, USA.

*Author for correspondence (gershont@neurology.unc.edu)

(D) T.R.G., 0000-0001-7034-6400
}

Received 25 April 2016; Accepted 16 September 2016 (ataxia telangiectasia and Rad3-related protein) mitigates proliferation-associated DNA damage (Brown and Baltimore, 2000) and has been implicated in microcephaly in Seckel syndrome (O'Driscoll et al., 2003). In mice, conditional deletion of Atr throughout the CNS during embryonic development similarly induces microcephaly, but particularly affects the cerebellum and ganglionic eminence (Lee et al., 2012). The precise mechanisms that cause DNA damage to impair growth specifically in the brain are unknown. We investigated the processes that restrict growth when Atr is deleted in the cerebellum in order to shed light on the pathogenesis of microcephaly associated with DNA damage and to determine whether the reliance of neural progenitors on ATR persists in progenitor-derived medulloblastoma.

Cerebellar growth depends on the postnatal proliferation of cerebellar granule neuron progenitors (CGNPs) in the external granular layer (EGL) along the outside of the cerebellum, which peaks between postnatal day (P) 5 and 7 (Hatten and Heintz, 1995). Although Atr deletion in nestin $(\mathrm{NES})^{+}$progenitors blocks cerebellar development prior to postnatal neurogenesis (Lee et al., 2012), the specific cellular processes restricting the growth of ATRdeficient progenitors have not been discerned. In vitro, ATR reduces DNA damage during proliferation by stabilizing stalled replication forks and by arresting the cell cycle to allow for DNA repair (Cimprich and Cortez, 2008). Replication fork collapse following ATR disruption produces DNA double-strand breaks (DSBs), which are considered the most toxic form of DNA damage (Couch et al., 2013). ATR also maintains S- and G2-phase checkpoints by phosphorylating CHK1 (CHEK1) in response to DNA damage (Smith et al., 2010). Determining how these functions combine in vivo to sustain neural progenitors is essential to understanding both the pathogenesis of Seckel syndrome and the requirement for ATR in brain development.

We found that ATR-deficient CGNPs continued to proliferate despite accumulating DNA damage, which induced populationwide, p53-dependent apoptosis. Blocking apoptosis in ATRdeficient CGNPs through co-deletion of the key apoptotic mediators Bax and Bak (Bakl) or co-deletion of p53 (Trp53) did not fully rescue the resulting cerebellar hypoplasia. Rather, premature cell cycle exit in Atr/Bax/Bak mutants or non-apoptotic cell death in Atr/p53 mutants redundantly limited CGNP population growth. Atr-deleted CGNPs also demonstrated diverse chromosomal abnormalities that were intensified by restriction of apoptosis. CGNPs are the cells of origin for sonic hedgehog (SHH) subgroup medulloblastomas; we found that Atr deletion blocked the tumorigenic effect of constitutive $\mathrm{SHH}$ activation in transgenic SmoM2 mice. These investigations define a crucial role for ATR in maintaining genomic integrity during brain development and suggest that ATR dependence might be exploited for medulloblastoma therapy. 


\section{RESULTS}

\section{Atr deletion induces CGNP apoptosis and cerebellar hypoplasia}

To analyze ATR function in CGNPs, we generated mice with conditional Atr deletion (Brown and Baltimore, 2003) in the MATH1 lineage. Math1 (Atoh1) is expressed by CGNPs in a rostrocaudal progression beginning at the anterior margins of the cerebellar cortex at embryonic day (E) 12.5 (Hatten and Roussel, 2011). We crossed the Math1-Cre and AtrloxP/loxP mouse lines to generate Math1-Cre;AtrloxP/loxP $\left(\right.$ Atr $\left.^{\text {M-cre }}\right)$ mice.

$A t r^{M-c r e}$ mice were viable and fertile, but by P12 displayed tremor and ataxia, suggesting impaired postnatal neurogenesis. These animals were born with hindbrains of normal appearance, with the EGL spread over the primordial cerebellum. By P3, however, the EGL in $A t r^{M-c r e}$ mice was noticeably thinner and less foliated than in Math1-Cre littermate controls with intact Atr (Math1-Cre; Atr $^{+/+}$or Math1-Cre;Atr $\left.{ }^{\text {loxP/+}}\right)$. Cerebellar hypoplasia in Atr ${ }^{M-c r e}$ mice became progressively more apparent with age (Fig. 1A).

We noted sparing of the posterior regions of the cerebellum in Atr $^{M-c r e}$ mice (Fig. 1A, middle row). To determine if this sparing was the result of incomplete recombination in these regions or was due to reduced dependence on ATR, we used $h G F A P$-Cre to delete Atr throughout the CGNP population. $h G F A P$-Cre mice express Cre recombinase in neural stem cells, targeting all cells of the cerebellum except the Purkinje cells (Andrae et al., 2001). In the resulting hGFAP-Cre;AtrloxP/loxP (Atr ${ }^{\text {G-cre }}$ ) mice, we found hypoplasia across the entire EGL. The EGL was thinned by P0 and completely lost by P7. These mice showed more severe tremor and died by P18 (Fig. 1A, bottom row; Fig. S1A,B).

To understand the cause of cerebellar hypoplasia in Atr mutants, we analyzed patterns of proliferation, DNA damage and apoptosis in $A t r^{M-c r e}$ and Atr ${ }^{G-c r e}$ mice by immunohistochemistry (IHC). Although prior studies showed that deletion of Atr in Nesexpressing progenitors blocks the prenatal proliferation of CGNP precursors (Lee et al., 2012), we observed no reduction in the postnatal proliferation of CGNPs in P3 Atr ${ }^{M-c r e}$ or P0 Atr ${ }^{G-c r e}$ cerebella, as demonstrated by the fraction of EGL cells expressing the mitosis marker phosphorylated histone H3 (pH3) (Fig. 1B,C, Fig. S1C) or the cycling cell marker proliferating cell nuclear antigen (PCNA) (Fig. S3D). However, we found significantly increased DNA damage as demonstrated by phosphorylated histone H2A.X ( $\gamma$ H2A.X), increased activation of the cellular DNA damage response as shown by phosphorylated ATM (p-ATM) and p53 (p-p53), and increased apoptosis as shown by the levels of cleaved caspase 3 (cC3) (Fig. 1B,C, Fig. S1C,D). Atr-deleted CGNPs therefore proliferate robustly, but accrue extensive DNA damage and undergo apoptosis.

\section{Deletion of Bax and Bak prevents cell death in Atr mutant CGNPs}

Extensive apoptosis complicated further analysis of ATR-deficient CGNPs. Prior studies showed that deletion of the apoptosis gatekeeper Bax is sufficient to stabilize CGNPs with radiationinduced or proliferation-associated DNA damage (Chong et al., 2000; Garcia et al., 2013; Williams et al., 2015). We found, however, that apoptosis of Atr-deleted CGNPs and cerebellar hypoplasia were not prevented by Bax co-deletion in Math1-Cre;AtrloxP/loxP; Bax loxP/loxP $\left(\right.$ Atr;Bax $\left.{ }^{\text {M-cre }}\right)$ animals (Fig. 2A, Fig. S2A,B). Codeletion of Atr and the Bax homolog Bak also failed to stabilize the CGNP population in Math1-Cre;AtrloxP/loxP $; \mathrm{Bak}^{-/-}\left(\right.$Atr;Bak $\left.{ }^{M-c r e}\right)$ mice (Fig. S2A,B). However, combining genetic deletion of both Bax and Bak in ATR-deficient mice (Math1-Cre;AtrloxP/loxP;
$B a x^{l o x P / l o x P} ; B^{-1-}$, or Atr;Bax;Bak $\left.k^{M-c r e}\right)$ effectively prevented CGNP apoptosis, partially rescuing cerebellar growth (Fig. 2A,B, Fig. S2A,C). TUNEL staining did not detect non-apoptotic cell death in Atr;Bax;Bak $k^{\text {M-cre }}$ cerebella that might have been missed by cC3 studies (Fig. 2B,D). These data show that Atr deletion in CGNPs, in contrast to other pro-apoptotic stimuli such as radiation, activated both BAX- and BAK-driven apoptosis.

We compared markers of proliferation, DNA damage and the DNA damage response in CGNPs of P3 Atr;Bax ${ }^{M-c r e}, A t r ; B a x ; B a k^{M-c r e}$ and Atr-intact Math1-Cre;Bax ${ }^{\text {loxP/loxP }} ; B^{-1} k^{-/} \quad\left(B a x ; B a k^{M-c r e}\right)$ controls by IHC. The fractions of $\mathrm{pH}^{+}$and $\mathrm{PCNA}^{+}$CGNPs were similar in Atr mutant and Atr-intact genotypes (Fig. 2D, Fig. S2C, Fig. S3C,D). CGNPs in Atr;Bax ${ }^{M-c r e}$ and Atr;Bax;Bak ${ }^{M-c r e}$ mice showed similar levels of $\gamma \mathrm{H} 2 \mathrm{~A}$.X. However, p-ATM, p-p53, and the cell cycle inhibitor p21 (CDKN1A) were all markedly increased in Atr;Bax;Bak ${ }^{\text {M-cre }}$ CGNPs (Fig. 2B,D, Fig. S2B,C). Apoptosisdisabled, ATR-deficient CGNPs were thus able to activate p53 without undergoing cell death.

\section{Atr and p53 double-mutant CGNPs undergo caspase- independent cell death}

To determine the functional significance of p53 activation in CGNPs of $A t r^{M-c r e}$ mice, we examined the phenotype of Math1Cre;AtrloxP/loxP $;$ p53 loxP/loxP $\left(\right.$ Atr;p53 $3^{M-\text { cre }}$ ) mice. $p 53$ deletion prevents CGNP apoptosis after radiation-induced DNA damage (Herzog et al., 1998). p53 deletion did not completely rescue cerebellar hypoplasia in Atr mutants (Fig. 2A, Fig. S3A), despite effective suppression of the p53 axis, as demonstrated by the absence of $\mathrm{p} 21$ and p53 activation (Fig. 2C,D). The fraction of $\mathrm{pH} 3^{+}$ and $\mathrm{PCNA}^{+}$CGNPs in Atr;p53 $3^{M-c r e}$ mice did not differ from Atrintact controls at P3 (Fig. 2D, Fig. S3B,D). Atr;p53 $3^{M-c r e}$ CGNPs accumulated significantly more DNA damage than Atr $^{M-c r e}$ or Atr; Bax;Bak ${ }^{M-c r e}$ CGNPs (Fig. 2D). This increased level of DNA damage did not trigger activation of caspase 3 (Fig. 2D). However, TUNEL staining demonstrated that cell death had occurred without caspase activation (Fig. 2C,D).

Atr; $53^{M-c r e}$ CGNPs showed abnormal morphologies that were different to the pyknotic nuclei found in the Atr ${ }^{\text {M-cre }}$ EGL. These features included giant multinucleated cells and cells with micronuclei (Fig. S3E), consistent with published descriptions of p53-deficient cancer cells undergoing necrosis after DNA damage (Vakifahmetoglu et al., 2008). To examine whether Atr;p53 $3^{\text {M-cre }}$ CGNPs were dying through regulated necrosis, we compared the phosphorylation of MLKL, an essential step in the necroptosis pathway (Cai et al., 2014). We found scattered CGNPs showing phosphorylated MLKL ( $\mathrm{p}-\mathrm{MLKL}$ ) in Math1-Cre, $53^{\text {M-cre }}$ and Atr; $p 53^{M-c r e}$ mice, but no increase in the Atr;p5 $53^{M-c r e}$ genotype (Fig. S3F). Thus, ATR/p53-deficient CGNPs undergo cell death without evidence of activating the apoptotic or necroptotic pathways.

\section{Accelerated cell cycle exit in Atr;Bax;Bak ${ }^{M-c r e}$ CGNPs}

To account for the incomplete rescue of cerebellar growth in Atr; Bax;Bak $k^{M-c r e}$ and Atr;p53 $53^{M-c r e}$ mice despite the prevention of CGNP apoptosis, we investigated CGNP proliferation over time. We compared the mitotic rate of Atr ${ }^{M-c r e}, A t r ; B a x ; B a k^{M-c r e}$ and Atr; $p 53^{M-c r e}$ CGNPs in the EGL with Atr-intact controls at P3, P5 and P7. We first determined that EGL mitotic rates did not vary significantly between the Atr-intact Math1-Cre, Bax;Bak ${ }^{M-c r e}$ and $p 53^{M-c r e}$ control genotypes, allowing us to pool controls (Fig. S3G). The mitotic rates of $A t r^{M-c r e}$ CGNPs did not differ from controls with any statistical significance at P3, P5 or P7: both groups showed 


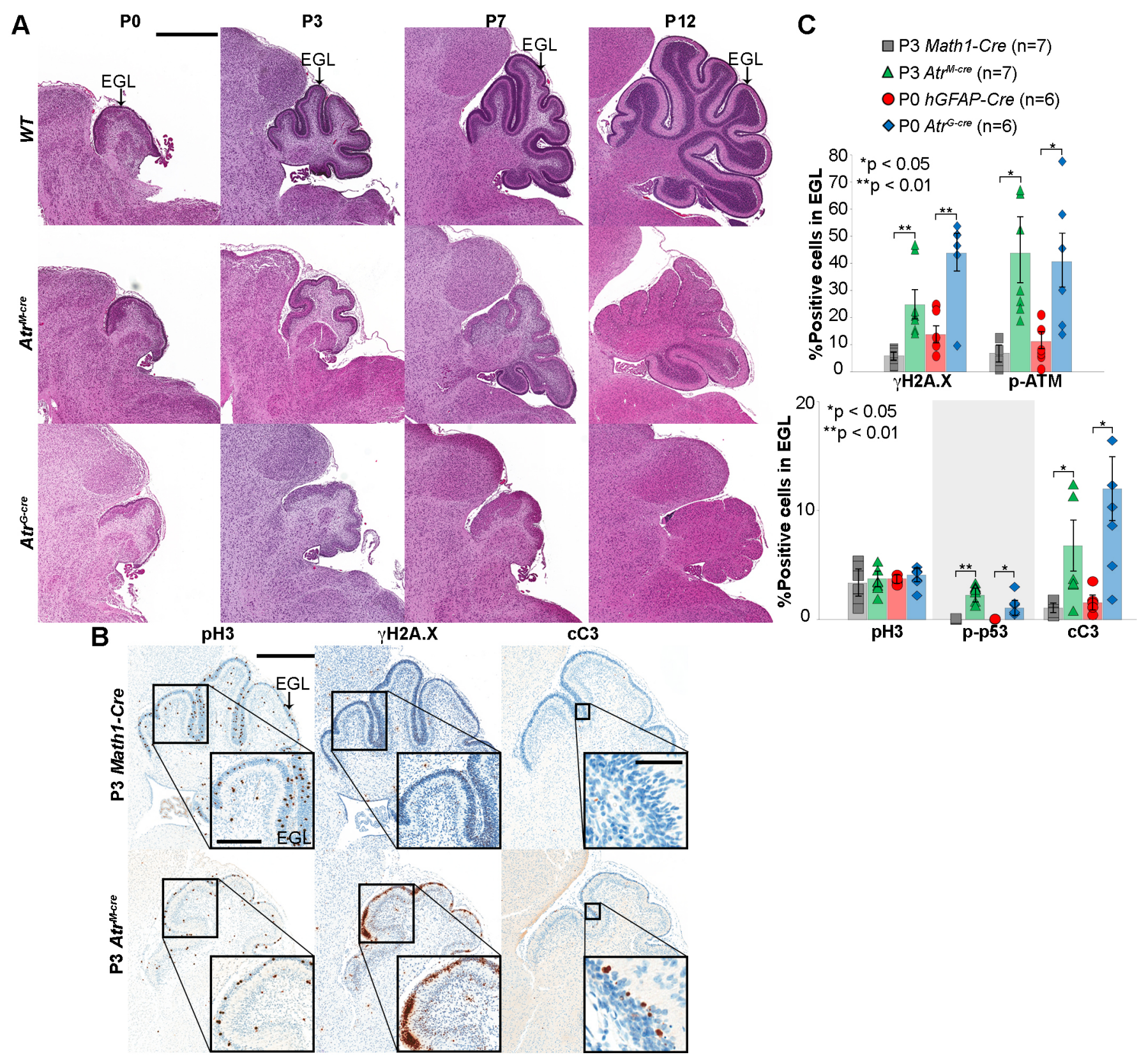

Fig. 1. Atr deletion in CGNPs induces postnatal DNA damage, p53 activation, apoptosis and cerebellar hypoplasia. (A) H\&E-stained sagittal brain sections from mice of the indicated genotypes and ages. (B) Sagittal cerebellar sections from P3 mice of the indicated genotypes stained for $\mathrm{pH} 3, \gamma \mathrm{H} 2 \mathrm{~A} . \mathrm{X}$ and cC3. (C) Quantification of replicate cerebella of the indicated genotypes, stained as in B and Fig. S1. Average is shown with s.e.m. $P$-values by two-sided independent samples $t$-test assuming unequal variances. All stainings were repeated on at least three biological replicates $(n)$. Scale bars: $800 \mu \mathrm{m}$ in $\mathrm{A} ; 400 \mu \mathrm{m}$ (main) and $200 \mu \mathrm{m}$ (inset) for $\mathrm{pH} 3,50 \mu \mathrm{m}$ for cC3 (inset) in B.

a small decrease in proliferation by P7 (Fig. 2E). By contrast, the proliferation rate of Atr;Bax;Bak ${ }^{M-c r e}$ CGNPs decreased markedly from P3 to P5 and from P5 to P7, and was significantly lower than in controls at both P5 and P7. These data show that non-proliferating cells were increased in the P5 and P7 EGL of Atr;Bax;Bak $k^{M-c r e}$ mice but not in $A t r^{M-c r e}$ mice with intact apoptosis. This increased cell cycle exit at P5 and P7 was p53 dependent, as the mitotic rate of apoptosis-disabled Atr;p53 $3^{\text {M-cre }}$ CGNPs was significantly higher than the Atr;Bax;Bak $k^{M-c r e}$ CGNP mitotic rate at both P5 and P7.

We noted clusters of cells in P20 Atr;Bax;Bak ${ }^{M-c r e}$ cerebella in the typically depopulated region where the EGL had been. These cells expressed the neuronal marker NEUN (RBFOX3) (Fig. 2F) and were $\mathrm{PCNA}^{-}$(data not shown), identifying them as differentiated neurons. The location of these neurons suggests that they derive from CGNPs, and similar ectopic neurons were not seen in Atr ${ }^{M-c r e}$, Atr;p53 $3^{M-c r e}$ or Atr-intact control cerebella. These observations provide evidence that premature, p53/p21-driven CGNP cell cycle exit after P3 limits the rescue effect of disabled apoptosis in Atr;Bax;Bak $k^{M-c r e}$ cerebella.

\section{Cell cycle checkpoint failure in ATR-deficient CGNPs with DNA damage}

ATR coordinates the cellular response to replication stress by activating cell cycle checkpoints (Abraham, 2001). Our studies of cerebellar sections indicated that Atr-deleted CGNPs continue to proliferate after developing detectable DNA damage. To assess 


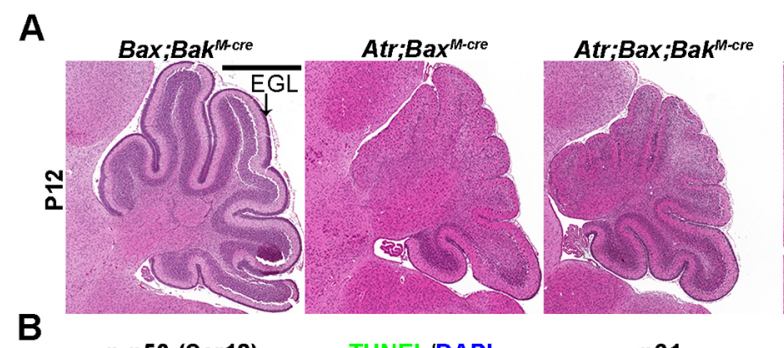

B
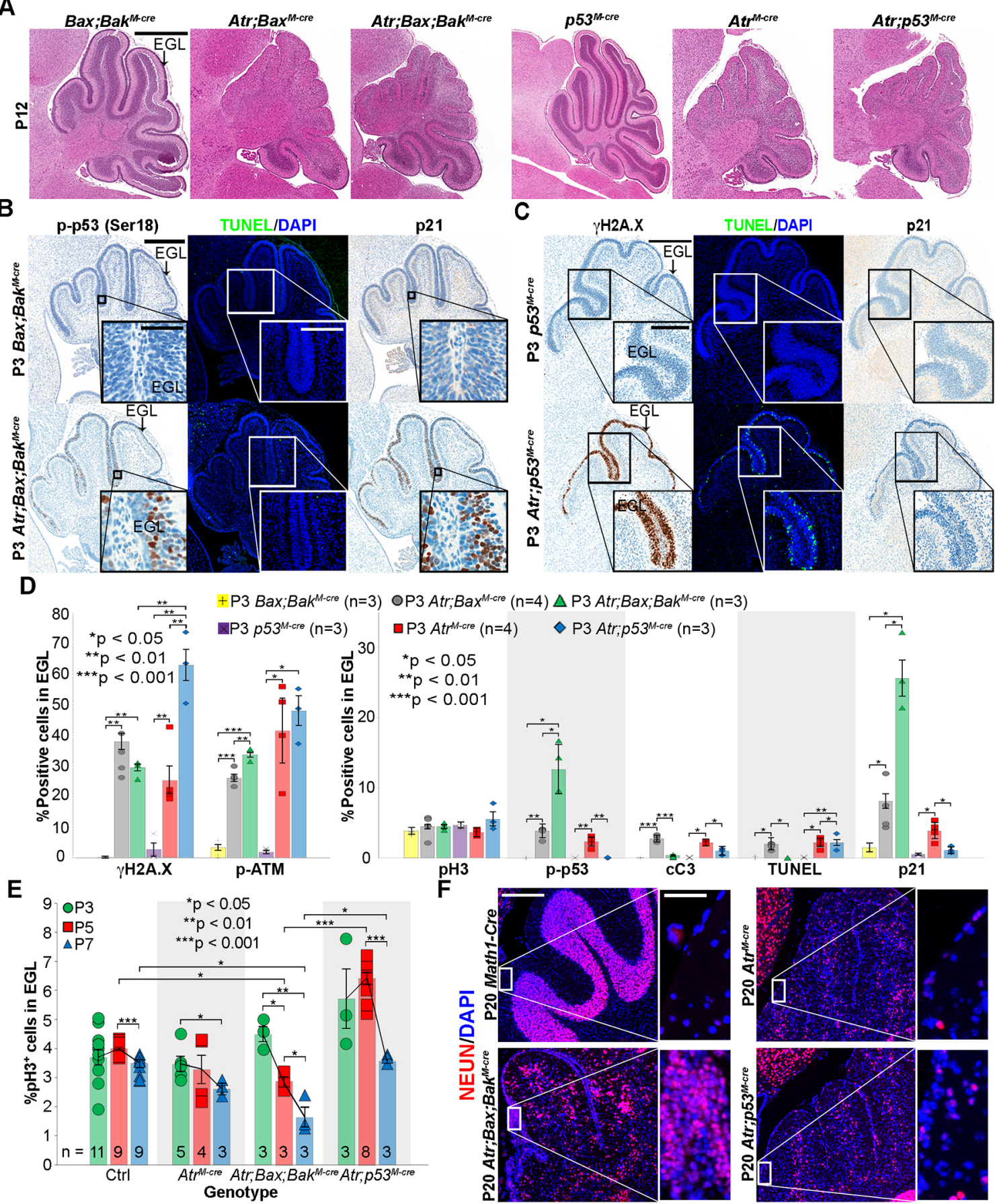

$(n=4) \triangle P 3$ Atr;Bax;Bak ${ }^{M-c r e}(n=3)$

4) $\diamond \mathrm{P} 3$ Atr;p53-cre $(\mathrm{n}=3)$
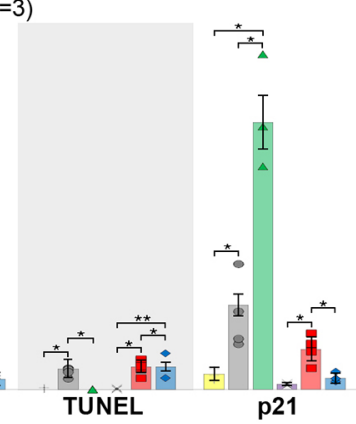

$\mathbf{F}$
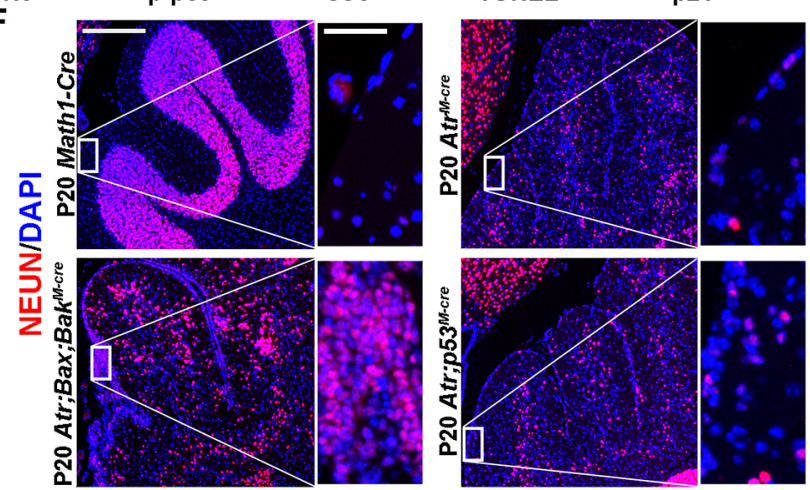

Fig. 2. Co-deletion of Bax and Bak, but not p53, blocks cell death in ATR-deficient CGNPs. (A) H\&E-stained, sagittal brain sections from P12 mice of the indicated genotypes, showing partial rescue of cerebellar development in Atr;Bax;Bak ${ }^{M-c r e}$ and Atr;p53 ${ }^{M-c r e}$ mice. (B) Sagittal cerebellar sections from P3 mice of the indicated genotypes stained for p-p53, TUNEL and p21. (C) Sagittal cerebellar sections from P3 mice of the indicated genotypes stained for $\gamma \mathrm{H} 2 \mathrm{~A}$.X,

TUNEL and p21. (D) Quantification of replicate cerebella of the indicated genotypes, stained as in B,C, Fig. S2 and Fig. S3. Bax;Bak ${ }^{M-c r e}$ and $p 53^{M-c r e}$ controls were not significantly different from the Math1-Cre controls presented in Fig. 1. (E) pH3 quantification in the external granular layer (EGL) of mice of the indicated genotypes at P3, P5 and P7. (F) NEUN staining on P20 sagittal cerebellar sections from mice of the indicated genotypes. Average with s.e.m. $P$-values by two-sided independent samples t-test assuming unequal variances. All stainings were repeated on at least three biological replicates ( $n$ ). Scale bars: $800 \mu \mathrm{m}$ in $\mathrm{A}$; $400 \mu \mathrm{m}$ (main) and $50 \mu \mathrm{m}$ (inset) for p-p53, $200 \mu \mathrm{m}$ for TUNEL (inset) in B; $400 \mu \mathrm{m}$ (main) and $200 \mu \mathrm{m}$ (inset) in C; $200 \mu \mathrm{m}$ (main) and $50 \mu \mathrm{m}$ (inset) in F.

checkpoint function in Atr mutants, we used fluorescence-activated cell sorting (FACS) to analyze freshly isolated CGNPs from the cerebella of P3 mice with and without Atr deletion, including Atr ${ }^{M-c r e}$, Atr;Bax;Bak ${ }^{M-c r e}$ and Atr;p53 $3^{M-c r e}$ knockout genotypes as well as Math1-Cre, Bax;Bak ${ }^{M-c r e}$ and $p 53^{M-c r e}$ controls. All cells were stained for DNA content (FxCycle Violet), $\gamma \mathrm{H} 2 \mathrm{~A} . \mathrm{X}$ and $\mathrm{pH} 3$.
Atr-deleted genotypes showed a strongly $\gamma \mathrm{H} 2 \mathrm{~A} . \mathrm{X}^{+}$ subpopulation that was not seen in controls (Fig. S4A). To generate Atr-intact CGNPs with comparable DNA damage, we subjected P3 wild-type (WT) mice to X-ray radiation (2 Gy) and then isolated and analyzed CGNPs $2 \mathrm{~h}$ later. These WT irradiated CGNPs showed $\gamma \mathrm{H} 2 \mathrm{~A}$.X staining that was comparable in intensity 
to the $\gamma \mathrm{H} 2 \mathrm{~A} . \mathrm{X}^{+}$CGNPs in Atr-deleted mice (Fig. S4A). DNA content staining effectively identified cells at G1, S and G2/M phases (Fig. S4A), and $\mathrm{pH} 3$ staining distinguished $\mathrm{G} 2$ from $\mathrm{M}$ phase (Fig. S4B). We found no statistically significant differences in cell cycle distribution between non-irradiated control genotypes (Fig. S4C), and these controls were pooled for further analysis.

Without $\gamma \mathrm{H} 2 \mathrm{~A}$.X selection, comparison of P3 CGNP cell cycle distribution between controls and Atr-deleted genotypes showed a significant difference only in the Atr;Bax;Bak ${ }^{M-c r e}$ CGNPs. These cells showed enrichment of G2 at the expense of G1 phase (Fig. 3A), consistent with either delayed progression or G2 arrest. However, comparing the $\gamma \mathrm{H} 2 \mathrm{~A} . \mathrm{X}^{+}$subsets from Atr-deleted genotypes and irradiated WT mice, we noted marked differences in M phase. All three Atr-deleted genotypes demonstrated CGNPs that were both $\gamma \mathrm{H} 2 \mathrm{~A} . \mathrm{X}^{+}$and $\mathrm{pH}^{+}$(Fig. 3B). By contrast, $\mathrm{pH}^{+}$ CGNPs were almost undetectable in the irradiated WT mice (Fig. 3B).

FACS results were confirmed by dual immunofluorescence (IF) for $\mathrm{pH} 3$ and $\gamma \mathrm{H} 2 \mathrm{~A}$.X or phospho-53BP1 (p-53BP1; 53BP1 is also known as TRP53BP1), another marker of DNA damage (Fig. 3C). First, in WT non-irradiated controls, $\mathrm{pH}^{+}$cells were always negative for $\gamma \mathrm{H} 2 \mathrm{~A} . \mathrm{X}$ and $\mathrm{p}-53 \mathrm{BP} 1$. Second, in irradiated WT mice, CGNPs that were $\gamma \mathrm{H} 2 \mathrm{~A} . \mathrm{X}^{+}$or $\mathrm{p}-53 \mathrm{BP} 1^{+}$were never $\mathrm{pH} 3^{+}$. Finally, in all Atr-deleted genotypes, $\gamma \mathrm{H} 2 \mathrm{~A} . \mathrm{X}^{+} / \mathrm{pH} 3^{+}$and $\mathrm{p}-53 \mathrm{BP} 1^{+} / \mathrm{pH} 3^{+}$ cells were common (Fig. 3C). The $\gamma \mathrm{H} 2 \mathrm{~A} . \mathrm{X}^{+} / \mathrm{pH}^{+}$CGNPs in Atrdeleted genotypes either entered $\mathrm{M}$ phase with DNA damage or persisted in $\mathrm{M}$ phase after acquiring DNA damage. These data do not conclusively show whether $\gamma \mathrm{H} 2 \mathrm{~A} . \mathrm{X}^{+}$, Atr-deleted CGNPs are able to complete mitosis. However, Atr deletion clearly permitted cells with DNA damage to be mitotic - a condition that is not seen in Atr-intact CGNPs.

Additionally, in each of the Atr-deleted genotypes, we found that $\gamma \mathrm{H} 2 \mathrm{~A} . \mathrm{X}^{+}$CGNPs were distributed across all phases of the cell cycle, with relative enrichment in S, G2 and M phases, compared with $\gamma \mathrm{H} 2 \mathrm{~A} . \mathrm{X}^{+}$CGNPs from irradiated WT mice (Fig. 3D). Consistent with these data, IF studies showed colocalization of $\gamma$ H2A.X with markers of G1 (cyclin D1), S (BrdU) and G2 (cyclin B1) phases (Fig. S4D) in Atr ${ }^{\text {M-cre }}$ cerebella. G1 depletion in Atrdeleted, $\gamma \mathrm{H} 2 \mathrm{~A} . \mathrm{X}^{+}$CGNPs could indicate either loss of H2A.X phosphorylation in G1 cells through DNA repair, or slowed return of $\gamma \mathrm{H} 2 \mathrm{~A} . \mathrm{X}^{+}$cells to G1. The abundance of $\gamma \mathrm{H} 2 \mathrm{~A} . \mathrm{X}^{+}$cells in each phase of the cell cycle, however, suggests that CGNPs with Atr deletion and DNA damage can progress through the cell cycle and, in the case of Atr;Bax;Bak ${ }^{M-c r e}$, have not all permanently arrested by P3. Altogether, at this single time point, most ATR-deficient CGNPs did not show evidence of impaired cell cycle function. However, Atr deletion increased the fraction of CGNPs with DNA damage and altered the barriers that normally prevent them from undergoing mitosis.

FACS data confirmed the increased cell death seen in Atr $^{M-c r e}$ and Atr;p53 $3^{M-c r e}$ CGNPs, which both showed increased sub-G1 fractions. We did not detect an increase in sub-G1 $\gamma \mathrm{H} 2 \mathrm{~A} . \mathrm{X}^{+}$ CGNPs in Atr;Bax;Bak ${ }^{M-c r e}$ cerebella (Fig. 3E). These increases in sub-G1 fractions are consistent with our observations of apoptotic cell death in the $A t^{M-c r e}$ genotype and non-apoptotic cell death in the Atr;p $53^{M-c r e}$ genotype.

\section{ATR protects CGNP chromosome integrity}

Our finding of impaired checkpoint function suggested that genomic fidelity might be impaired in Atr-deleted CGNPs. In vitro studies have shown incomplete DNA replication and chromosomal fragmentation in Atr mutant cells (Brown and
Baltimore, 2000). To determine if chromosomal damage accumulates in ATR-deficient cells in vivo and to quantify the damage, we karyotyped Atr-deleted CGNPs freshly harvested from P3 cerebella. We dissociated CGNPs from Atr ${ }^{M-c r e}$, Atr;Bax; Bak $k^{M-c r e}$ and Atr;p5 $3^{M-c r e}$ mice and Math1-Cre, Bax;Bak $k^{M-c r e}$ and $p 53^{M-c r e}$ controls. Dissociated cells were treated ex vivo with Colcemid, and metaphase spreads were analyzed by conventional karyotyping and spectral karyotyping (SKY). We noted frequent chromosome breaks in all Atr mutant genotypes and a spectrum of abnormalities, including dicentric chromosomes, end-to-end fusions, deletions, translocations, and whole chromosome gains or losses (Fig. 4A). In addition, Atr;p53 $3^{M-c r e}$ CGNP chromosomes showed segregation defects ( premature sister chromatid separation). These abnormalities were not detected in controls (Atr ${ }^{M-c r e} 35 \%$, $P<0.01 ;$ Atr $;$ Bax $;$ Bak $k^{M-c r e} 48 \%, P<0.01 ;$ Atr; $53^{M-\text { cre }} 49 \%, P<0.01$; Student's $t$-test).

For statistical analysis, we divided metaphases with varying degrees of chromosomal damage into four bins: 1-10 chromosome breaks/cell; $>10$ chromosome breaks/cell and $<50 \%$ chromosomes fragmented (mild); $>10$ chromosome breaks/cell, $>50 \%$ chromosomes fragmented, and some preserved chromosome morphology (moderate); and $>10$ chromosome breaks/cell, $>50 \%$ chromosomes fragmented, and complete loss of chromosome morphology (severe) (Fig. 4B). In all Atr-deleted genotypes, the frequencies of cells with all degrees of chromosomal damage were increased, but the increases in $A t r^{M-c r e}$ cells with moderate and severe damage were not statistically significant. Moderate and severe damage, however, were particularly enriched in the apoptosis-incompetent Atr;Bax;Bax ${ }^{M-c r e}$ and Atr;p53 $3^{M-c r e}$ genotypes (Fig. 4C). These data show that CGNPs require ATR to maintain chromosomal integrity during proliferation, and that apoptosis limits the accumulation of genetic abnormalities by removing damaged cells from the population. Karyotype analyses also reaffirm that CGNPs with damaged DNA progress to M phase in Atr mutants.

\section{Transcriptomic adaptations to ATR deficiency are predominantly $\mathbf{p 5 3}$ driven}

We used RNA-Seq to determine the transcriptomic alterations induced by proliferation-associated DNA damage in the absence of Atr. Despite a wide body of literature on the cellular consequences of ATR loss, the impact on the transcriptome had not previously been reported. The prolonged survival of Atr-deleted CGNPs in Atr; Bax;Bax ${ }^{M-c r e}$ and Atr;p $53^{M-c r e}$ mice allowed us to analyze mRNA abundance without losing cells with DNA damage from the population through cell death. We isolated and purified total RNA for RNA-Seq analysis from the cerebella of apoptosis-incompetent, ATR-deficient P3 mice (Atr;Bax;Bak ${ }^{M-c r e}$ and Atr;p53 $3^{M-c r e}$ ) and corresponding age-matched controls ( $B a x ; B a k^{M-c r e}$ and $p 53^{M-c r e}$ ). The abundance of mRNAs may be altered by changes in either production or degradation. ATR has been implicated in RNA processing (Chandris et al., 2010), and Atr deletion may alter both gene transcription and mRNA stability. Our data thus identify transcriptomic, rather than transcriptional changes.

We noted that Atr deletion evoked a richer, more complex pattern of transcriptomic changes in the Bax/Bak co-deleted background compared with the $p 53$ co-deleted background. Principal component analysis (PCA) demonstrated that the second component of variation explained $16 \%$ of variance in global gene expression, and separated Atr;Bax;Bak $k^{M-c r e}$ from Bax;Bak ${ }^{M-c r e}\left(P=4.41 \times 10^{-7}\right.$, Hotelling's $t$-square test) but not Atr;p53 $3^{M-c r e}$ from $p 53^{M-c r e}(P=0.20$, Hotelling's $t$-square test) (Fig. 5A). This difference in separation 
A

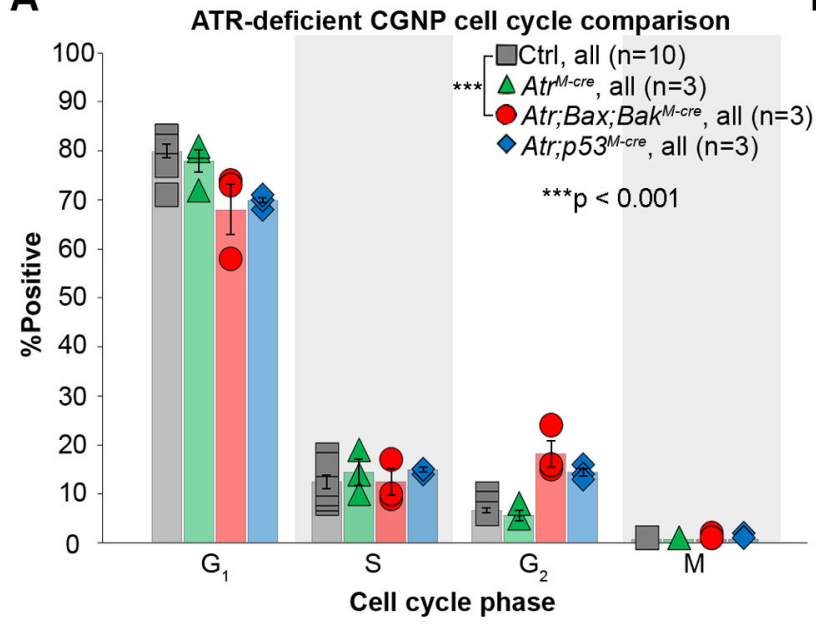

C
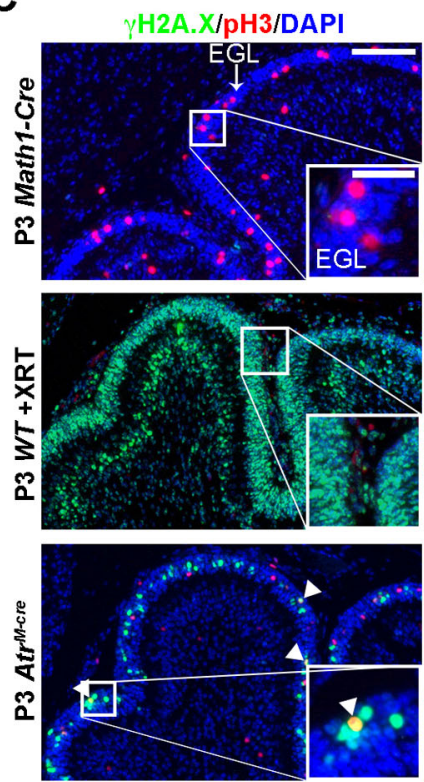

$\mathbf{E}$

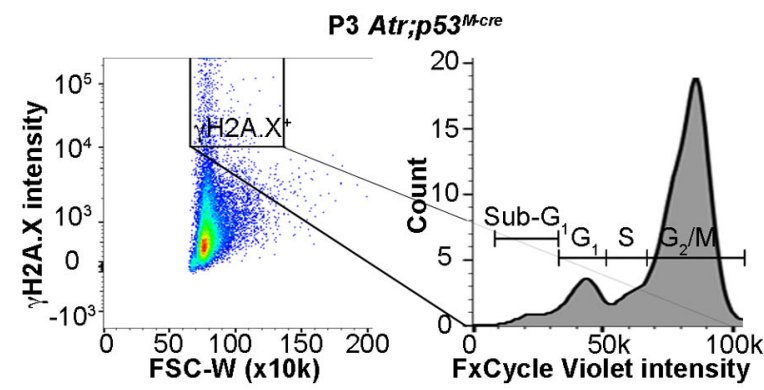

$\mathrm{p}-53 \mathrm{BP} 1 / \mathrm{pH} 3 / \mathrm{DAPI}$
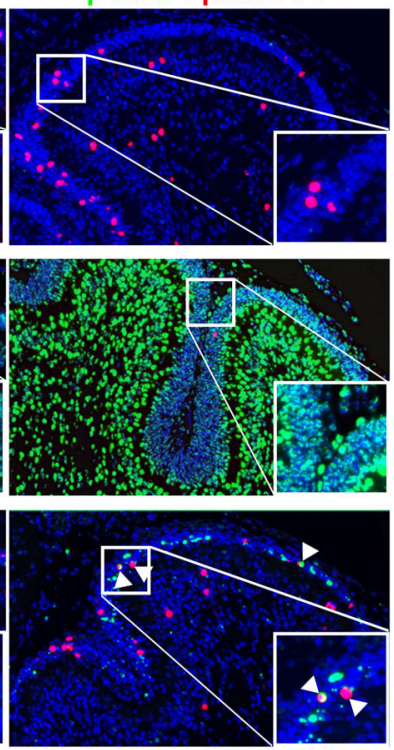

FxCycle Violet intensity
B

$\square W T+X R T, \gamma H 2 A . X^{+}(n=3)$
$\triangle A t{ }^{M-c r e}, \gamma H 2 A . X^{+}(n=3)$
Atr;BaX;Bak ${ }^{M-c r e}, \gamma H 2 A . X^{+}(n=3)$
$\triangle$ Atr;p533-cre, $\gamma H 2 A . X^{+}(n=3)$

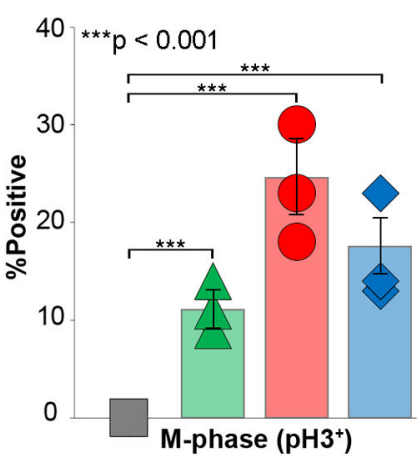

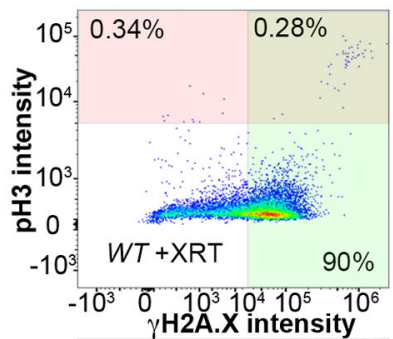

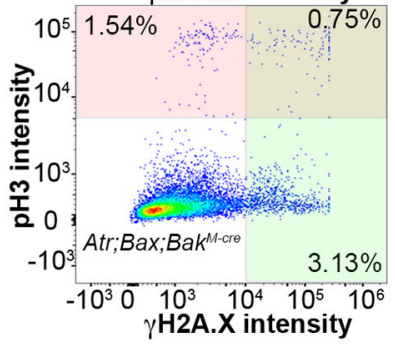

D

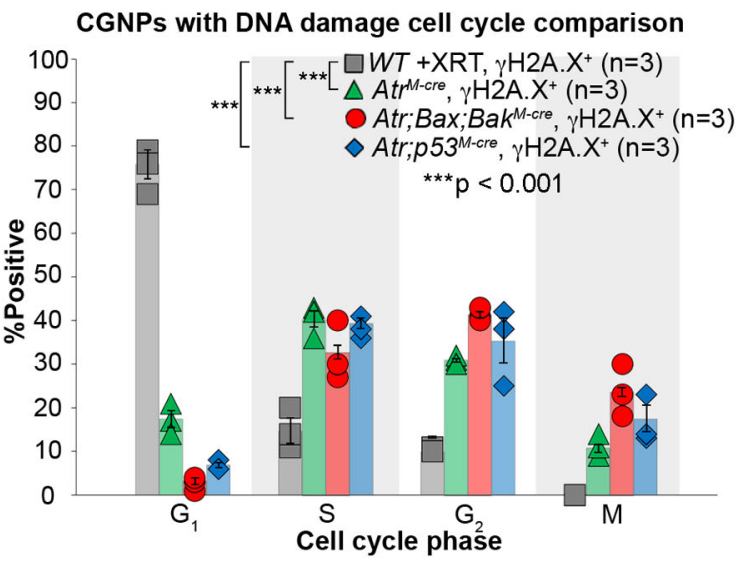

ATR-deficient CGNPs with Sub-G 1 DNA content

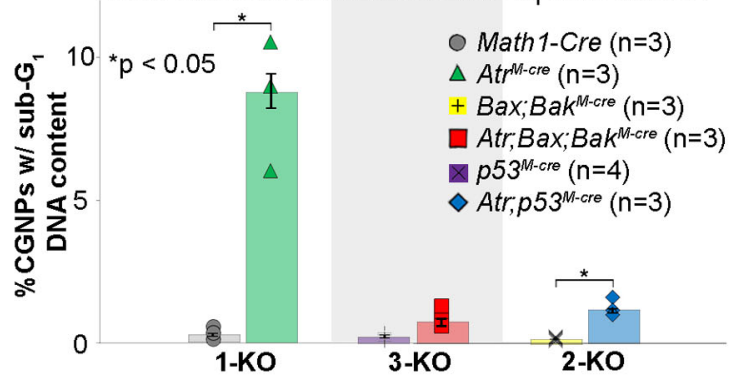

Fig. 3. Increased DNA damage and inappropriate mitotic entry in Atr-deleted CGNPs. (A) FACS analysis without subsetting for $\gamma \mathrm{H} 2 \mathrm{~A}$.X on CGNPs from P3 mice of the indicated genotypes. M phase was determined by $\mathrm{pH} 3$ labeling. $P$-values by chi-square test. (B) Quantification of $\gamma \mathrm{H} 2 \mathrm{~A}$. $\mathrm{X}^{+} / \mathrm{pH} 3^{+} \mathrm{CGNPs}$ from P3 mice of the indicated genotypes. $P$-values by two-sided independent samples $t$-test assuming unequal variances. (C) Double labeling for $\gamma \mathrm{H} 2 \mathrm{~A}$. $\mathrm{X} / \mathrm{pH} 3$ and $\mathrm{p}-53 \mathrm{BP} 1 / \mathrm{pH} 3$ on sagittal cerebellar sections from P3 mice of the indicated genotypes. Arrowheads indicate double positives. Scale bars: $100 \mu \mathrm{m}$ (main) and $50 \mu \mathrm{m}$ (inset). (D) FACS analysis with subsetting for $\gamma \mathrm{H} 2 \mathrm{~A} . \mathrm{X}^{+}$CGNPs from P3 mice of the indicated genotypes. $P$-values by chi-square test. (E) Quantification of 'sub-G1' DNA content detected by FACS in CGNPs from mice of the indicated genotypes. $P$-values by two-sided independent samples $t$-test assuming unequal variances. Average with s.e.m. All stainings were repeated on at least three biological replicates $(n)$.

on PCA indicates that $p 53$-deleted CGNPs are more restricted in their transcriptomic response to ATR deficiency. Consistent with reduced transcriptional responsiveness with Atr/p53 co-deletion, the number of genes differentially expressed in Atr;Bax;Bak $k^{\text {-cre }}$ cerebella compared with controls was significantly greater than in Atr;p $53^{M-c r e}$ cerebella compared with controls $\left(P=2.8 \times 10^{-52}\right.$, Fisher's exact test $)$. 
A

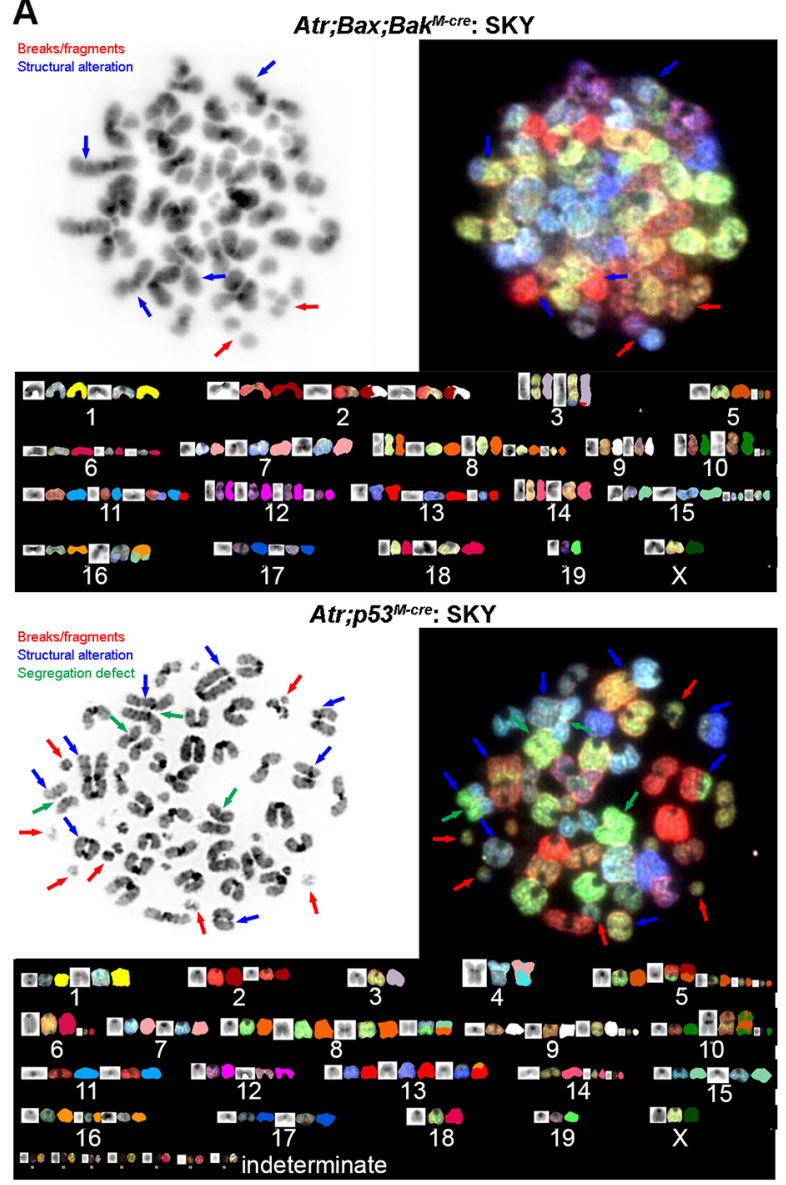

B
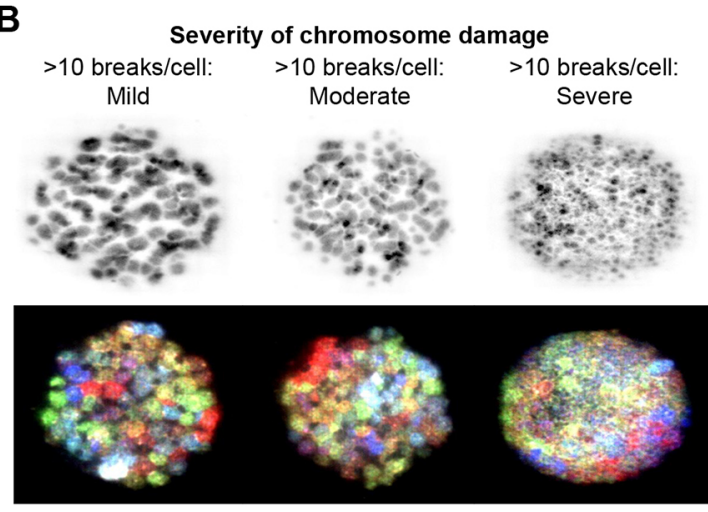

C

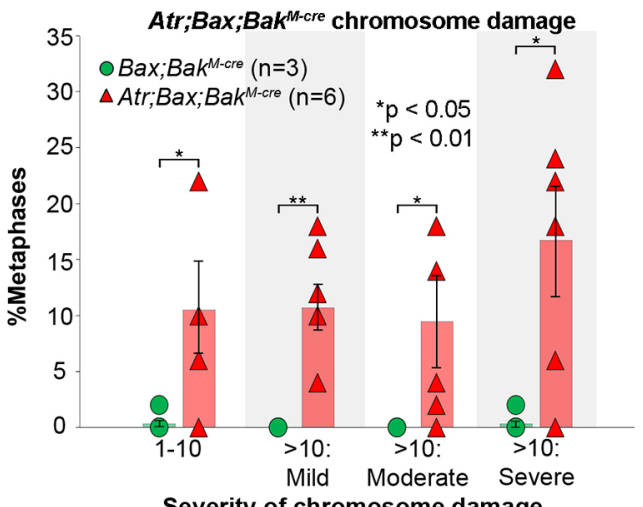

Severity of chromosome damage (Number of chromosome breaks/cell)

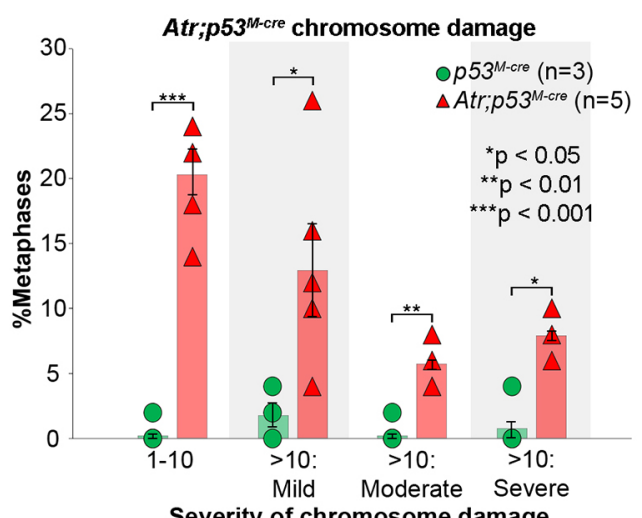

Severity of chromosome damage (Number of chromosome breaks/cell)

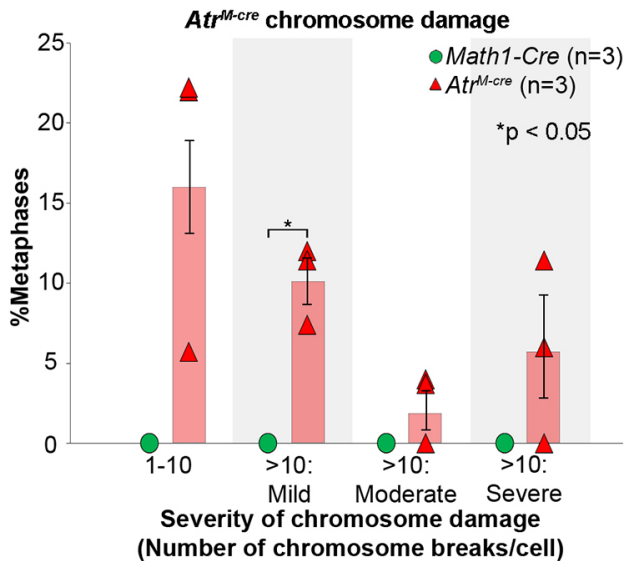

Fig. 4. Atr deletion compromises the chromosome integrity of proliferating CGNPs. (A) Metaphase chromosome spread and SKY analysis on Atr;Bax; $B a k^{M-c r e}$ and Atr;p53 ${ }^{M-c r e}$ CGNPs. (B) Severity of chromosome damage in CGNPs with $>10$ chromosome breaks/cell, grouped into mild, moderate and severe. (C) The distribution of CGNPs with chromosomal abnormalities by genotype. Average with s.e.m. $P$-values by two-sided independent samples $t$-test assuming unequal variances. $n$, number of biological replicates, with 35-50 metaphases counted per replicate.

Using a false discovery rate (FDR) of $<0.01$ and considering only genes meeting a fold-change (FC) criterion of $\mid \log _{2}$ (knockout/ control) $\mid>1.5$, we identified 339 genes differentially expressed by ATR-deficient cells in the Bax/Bak-deleted background, as compared with 56 genes differentially expressed by ATR-deficient cells in the $p 53$-deleted background, with nine genes represented in both sets (Fig. 5B, Tables S1 and S2). This overlap demonstrates a high degree of correspondence between the gene sets $\left(P=2.0 \times 10^{-8}\right.$, hypergeometric test). However, $P$-values for differentially expressed genes were consistently smaller in the p53-deleted comparison, as visualized in plots of FC versus significance (Fig. 5C), indicating an overall smaller transcriptomic effect of Atr deletion in the absence of $p 53$. Expression microarray analysis comparing mRNA from Atr;Bax;Bak $k^{M-c r e}$ versus Bax;Bak $k^{M-c r e}$ cerebella defined a gene set that corresponded well with the differential gene set defined by RNA-Seq (Fig. S5A). By contrast, microarray analysis was unable to identify any differentially expressed genes in the Atr;p53 $3^{M-c r e}$ versus $p 53^{M-c r e}$ comparison, consistent with an overall low signal-to-noise ratio in the absence of $p 53$. 
A

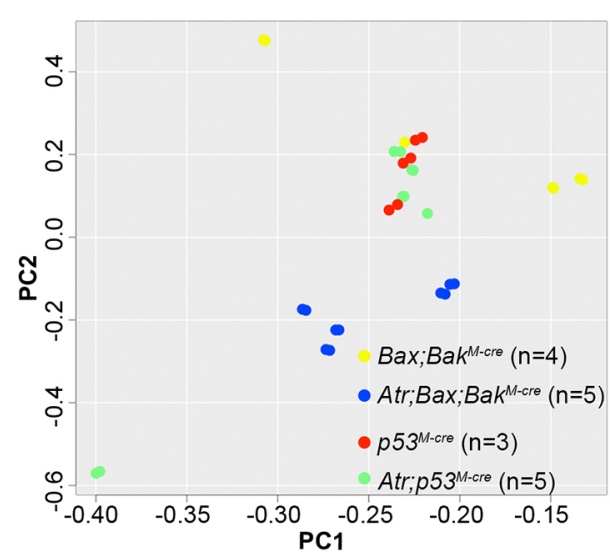

C

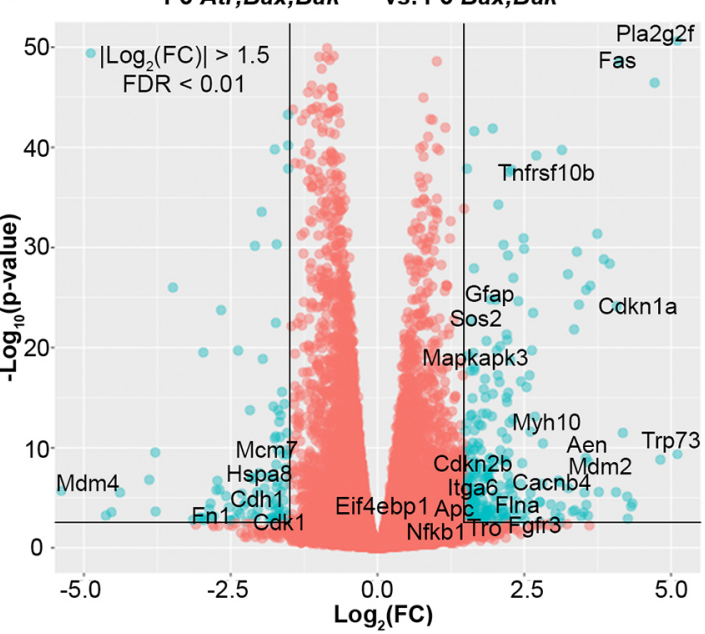

D
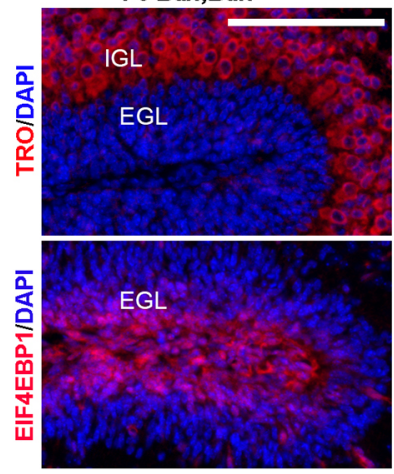

B

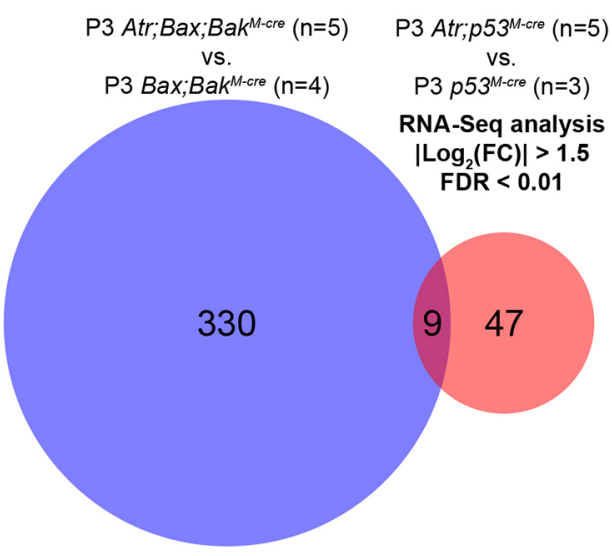

P3 Atr;p53 $3^{M-c r e}$ vs. P3 p53 $3^{M-c r e}$

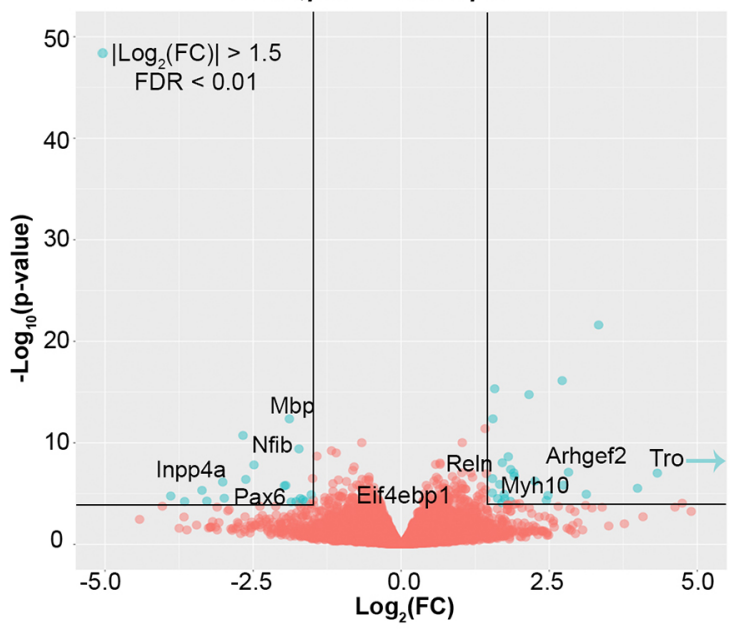

P3 $p 53^{M-c r e}$

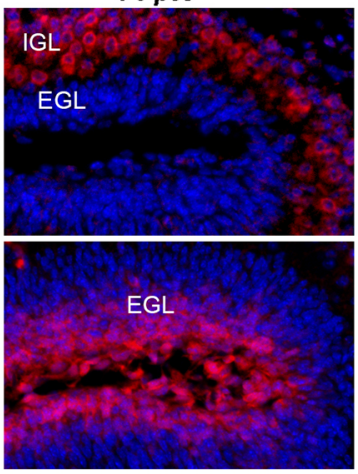

P3 Atr;p53 ${ }^{\text {M-cre }}$

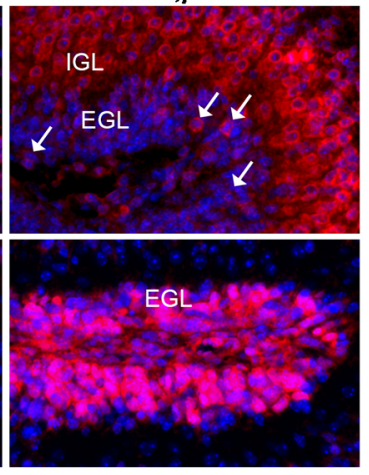

Fig. 5. The p53 pathway controls the CGNP transcriptomic response to Atr deletion. (A) PCA on gene expression of CGNPs from P3 mice of the indicated genotypes, as determined by RNA-Seq. (B) Differential gene expression analysis of RNA-Seq data using the indicated criteria comparing mice of the indicated genotypes. (C) Volcano plots of $\log _{2}(F C)$ versus $-\log _{10}\left(P\right.$-value) for Atr;Bax;Bak ${ }^{M-c r e}$ compared with Bax;Bak ${ }^{M-c r e}$ and for Atr;p53 $3^{M-c r e}$ compared with $p 53^{M-c r e}$, with color threshold (teal dots) set at $\log _{2}(F C)>1.5$ and FDR $<0.01$ (lines). (D) IF for TRO and EIF4EBP1 on sagittal cerebellar sections from P3 mice of the indicated genotypes. Arrows indicate TRO ${ }^{+}$cells. Scale bar: $100 \mu \mathrm{m}$. IGL, internal granular layer. All stains were repeated on at least three biological replicates $(n)$.

Pathway analysis of the 339 genes differentially expressed in Atr; Bax;Bax ${ }^{\text {M-cre }}$ CGNPs demonstrated a strong correspondence between these genes and the molecular signature of p53dependent transcription. Activation of p53 can induce both apoptosis and cell cycle arrest (Zuckerman et al., 2009), and we noted that regulators of both processes were enriched in the Atr;Bax; Bax ${ }^{M-c r e}$ gene set (Table 1). By contrast, we did not identify any pattern in Atr;p53 $3^{M-c r e}$ CGNPs that could be attributed to a specific transcriptional regulator (Table S3) and, consistent with the lack of increased MLKL activation, we found no elevation of necroptosisassociated genes such as RIP kinases.

We used IF to determine the protein expression patterns of trophinin (TRO), which was among the nine genes identified as differentially expressed in both Atr-deleted genotypes, and for which we were able to obtain effective antibodies. Examining $B a x ; B a k^{M-c r e}$ and $p 53^{M-c r e}$ control brains, we found that TRO was expressed in all differentiated neurons of the brain, but absent in progenitor regions (Fig. 5D). In Atr-deleted Atr;Bax;Bax ${ }^{M-c r e}$ and Atr;p53 $3^{M-c r e}$ 
Table 1. Pathway enrichment analysis on significantly differentially expressed genes between CGNPs from P3 Atr;Bax;Bak ${ }^{M-c r e}$ and Bax;Bak ${ }^{M-c r e}$ cerebella

\begin{tabular}{|c|c|c|c|c|}
\hline Database & Pathway & $\begin{array}{l}\text { Fold } \\
\text { enriched }\end{array}$ & FDR & Genes \\
\hline \multirow[t]{3}{*}{$\begin{array}{l}\text { Kyoto Encyclopedia of Genes and } \\
\text { Genomes }\end{array}$} & p53 signaling pathway & 27 & $5.9 \mathrm{E}-8$ & $\begin{array}{l}\uparrow C d k n 1 a, \uparrow F a s, \uparrow M d m 2, \uparrow \text { Pmaip } 1, \uparrow \text { Sesn2, } \\
\quad \uparrow T r p 73, \downarrow C d k 1, \downarrow M d m 4\end{array}$ \\
\hline & Pathways in cancer & 7 & $1.4 \mathrm{E}-3$ & $\begin{array}{l}\uparrow C d k n 1 a, \uparrow \text { Fas, } \uparrow F g f r 3, \uparrow \text { ltga6 }, \uparrow M d m 2, \uparrow \text { Sos } 2, \\
\downarrow A p c\end{array}$ \\
\hline & MAPK signaling pathway & 9 & $1.1 \mathrm{E}-5$ & $\begin{array}{l}\uparrow \text { Cacnb4, } \uparrow \text { Fas, } \uparrow F g f r 3, \uparrow \text { Mapkapk3, } \uparrow \text { Pla2g2f, } \\
\uparrow \text { Sos2, } \downarrow \text { Hspa8 }\end{array}$ \\
\hline \multirow{2}{*}{ Wiki Pathways* } & $\begin{array}{l}\text { miRNA regulation of DNA damage } \\
\text { response }\end{array}$ & 24 & $6.0 \mathrm{E}-7$ & $\begin{array}{l}\uparrow C d k n 1 a, \uparrow \text { Fas, } \uparrow M d m 2, \uparrow \text { Pmaip } 1, \uparrow \text { Tnfrsf10b, } \\
\downarrow \text { Cdk1, } \text { Mcm }^{2}\end{array}$ \\
\hline & G1 to S cell cycle control & 14 & $1.4 \mathrm{E}-3$ & $\uparrow C d k n 1 a, \uparrow M d m 2, \downarrow C d k 1, \downarrow M c m 7$ \\
\hline \multirow[t]{2}{*}{ Gene Ontology Biological Process ${ }^{\ddagger}$} & p53 signal transduction & 15 & $1.7 \mathrm{E}-4$ & $\begin{array}{l}\uparrow C d k n 1 a, \uparrow M d m 2, \uparrow \text { Pmaip1, } \uparrow \text { Sesn2, } \uparrow \text { Trp53inp1, } \\
\quad \downarrow M d m 4\end{array}$ \\
\hline & Cell cycle & 4 & 4.4E-4 & $\begin{array}{l}\uparrow C d k n 1 a, \uparrow M d m 2, \uparrow T r p 53 i n p 1, \downarrow A n a p c 1, \downarrow C d k 1, \\
\quad \downarrow M c m 7\end{array}$ \\
\hline
\end{tabular}

Differentially expressed genes are defined as $\log _{2}(\mathrm{FC})>1.5$ and FDR<0.01. Subset by consensus between RNA-Seq and microarray. Arrows indicate whether an individual gene within that pathway is upregulated or downregulated.

*Accessed via WebGestalt.

${ }^{\ddagger}$ Accessed via Panther.

$\S$ Also as determined by KEGG accessed via EnrichNet.

cerebella, however, TRO was detected within the CGNP layer (EGL), indicating upregulation of the protein as well as the RNA.

From the larger set of 124 genes that were upregulated in the Atr mutant cerebella of both p53-deleted and Bax/Bak-deleted backgrounds with $\mathrm{FDR}<0.01$, irrespective of $\mathrm{FC}$, we selected Eif4ebp1 for further analysis. EIF4EBP1 is known to regulate protein translation downstream of mTOR (Fingar et al., 2002), a PIKK kinase homologous to ATR (Lovejoy and Cortez, 2009). Atrdeleted CGNPs demonstrated marked upregulation of EIF4EBP1 protein. In control cerebella, EIF4EBP1 was limited to a subset of CGNPs at the outer margin of the EGL, whereas in Atr-deleted cerebella EIF4EBP1 was detected throughout the EGL (Fig. 5D). Our in situ IF data, along with our microarray results, thereby validate our RNA-Seq findings.

We further examined our RNA-Seq data to determine whether Atr deletion produced recurrent point mutations or other changes in RNA sequence. We did not detect a significant change in mismatch rate associated with Atr deletion (Fig. S5B). Fusion transcript detection software suggested a small number of fusion products, only one of which, with unknown biological function, emerged with statistical significance (Fig. S5C). Together with our karyotype analysis, these data suggest that, although Atr deletion significantly altered chromosome structure, it did not substantially increase the rate of point mutations or recurrent fusion transcripts.

\section{ATR is required for medulloblastoma tumorigenesis}

Prior studies have shown that deletion of diverse DNA repair pathway genes, combined with p53 deletion, induces medulloblastoma in mice (Frappart et al., 2007, 2009; Holcomb et al., 2006; Lee and McKinnon, 2002). To determine if Atr mutation predisposes mice to medulloblastoma, we followed the viability and neurologic function of $12 \mathrm{Atr} ; p 53^{\mathrm{M} \text {-cre }}$ and $12 \mathrm{Atr} ; \mathrm{Bax}$; $B a k^{M-c r e}$ mice for more than 300 days (supplementary Materials and Methods). These mice showed no progressive neurological changes or other evidence of tumorigenesis, and their cerebella remained hypoplastic (data not shown). The absence of tumors despite impaired genomic stability and p53 deletion suggests that medulloblastoma tumorigenesis, like postnatal neurogenesis, might require ATR function.

We directly tested this suggested requirement for ATR in medulloblastoma tumorigenesis by deleting Atr in tumor-prone hGFAP-Cre;SmoM2 mice (Schuller et al., 2008). CGNPs in SmoM2 ${ }^{G-c r e}$ mice with intact Atr gave rise to medulloblastoma with $100 \%$ frequency by $\mathrm{P} 7$, causing the mice to die from tumor progression by P20 (Fig. 6A). By contrast, Atr deletion in SmoM2; Atr ${ }^{G-c r e}$ animals completely blocked tumor formation, as seen and quantified by Hematoxylin and Eosin (H\&E) staining and by IF for PCNA, which marks proliferating tumor cells (Fig. 6A). The inability of Atr-deleted CGNPs in SmoM2;Atr ${ }^{G-c r e}$ mice to give rise to tumors suggests that ATR inhibition might effectively restrict medulloblastoma growth.

\section{ATR inhibitor administered in vivo induces DNA damage specifically in CGNPs}

To determine whether acute disruption of ATR function during postnatal neurogenesis in WT mice would recapitulate conditional Atr deletion, we developed a novel formulation of the small molecule ATR inhibitor VE-822 (pVE-822) (Charrier et al., 2011; Fokas et al., 2012). We first demonstrated that VE-822 induces DNA damage and apoptosis in isolated CGNPs in vitro in a timeand dose-dependent manner (Fig. S6A). To enhance delivery of VE-822 across the blood-brain barrier, we encapsulated VE-822 in poly(2-oxazoline) micelles, generating pVE-822 (Fig. S6B,C). We found that pVE-822, administered by intraperitoneal (IP) injection, induced $\gamma \mathrm{H} 2 \mathrm{~A}$.X and $\mathrm{cC} 3$ in the EGL, without affecting the surrounding differentiated neurons in the cerebellum or cortical neurons (Fig. 6B,C). As with Atr deletion, acute ATR inhibition by pVE-822 promoted upregulation of EIF4EBP1 in CGNPs, demonstrating that EIF4EBP1 is an effective biomarker of ATR disruption (Fig. 6D). These results show that pVE-822 effectively crosses the blood-brain barrier in bioactive concentrations after IP administration, and that acute, global loss of ATR function specifically disrupts CGNPs. The absence of neuronal toxicity associated with pVE- 822 administration is 

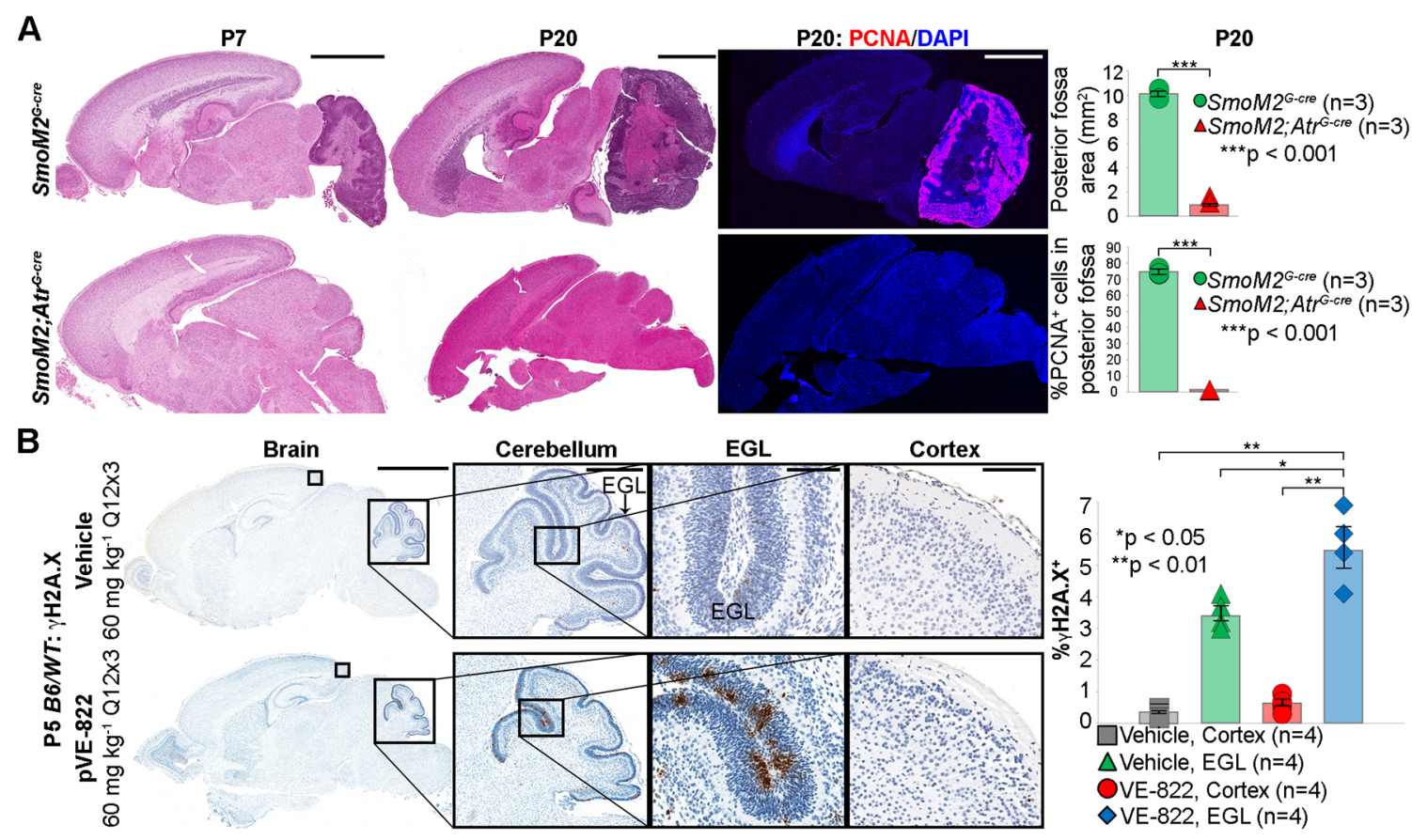

Cerebellum

EGL

Cortex

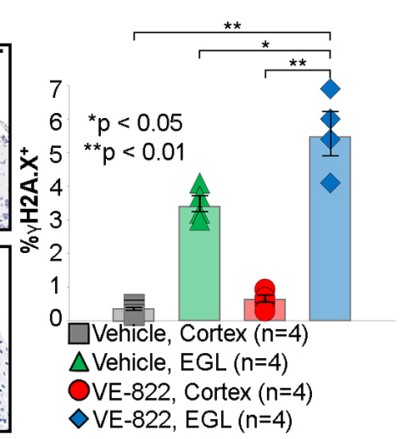

C
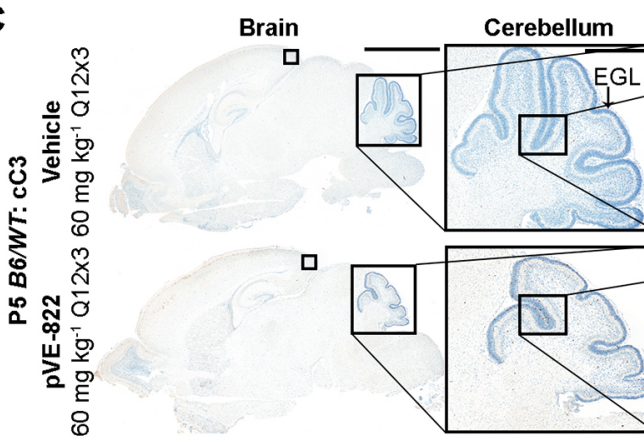

EGL

Cortex
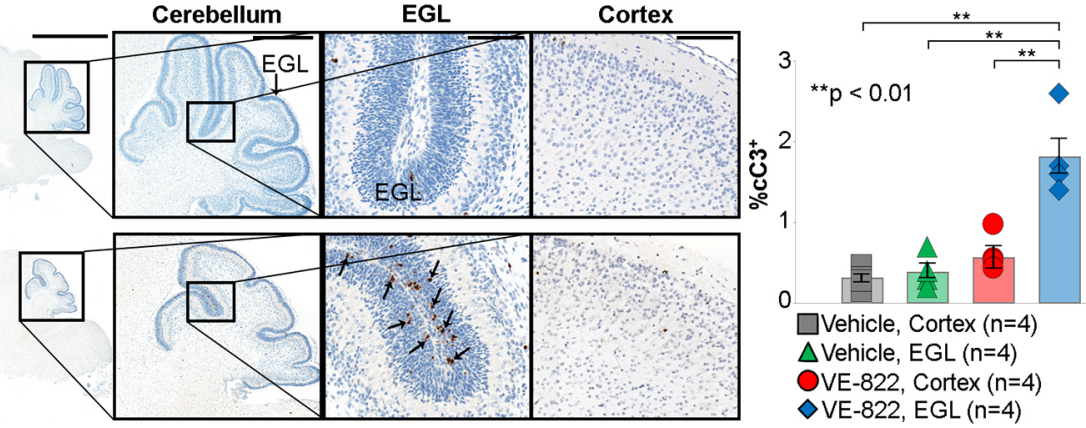

\section{D}
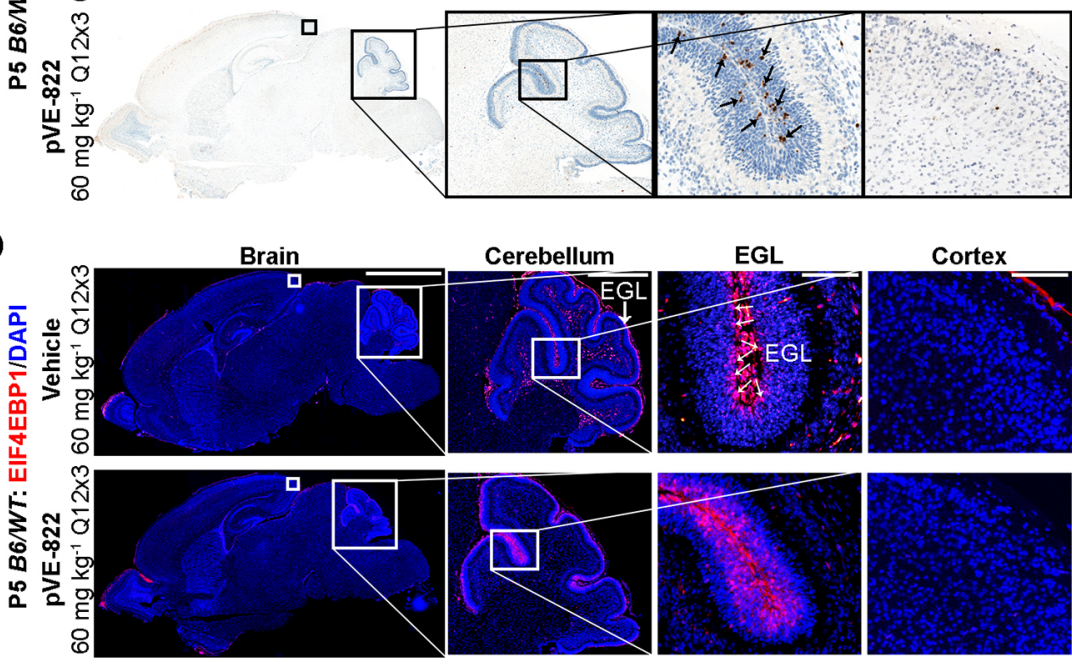

$\checkmark V E-822, E G L(n=4)$

Fig. 6. Atr deletion blocks medulloblastoma tumorigenesis, and acute in vivo ATR inhibition induces DNA damage and apoptosis in the postnatal cerebellum. (A) Sagittal H\&E- and PCNA-stained brain sections at P7 and P20 show the absence of tumor formation in SmoM2;AtrG-cre mice. Bar charts compare total area and percentage $\mathrm{PCNA}^{+}$cells in the P20 posterior fossa by genotype. (B-D) Sagittal sections of whole brains, cerebella and cortices from P5 WT mice injected with pVE-822 or vehicle, stained for (B) $\gamma \mathrm{H} 2 A$.X, (C) cC3 or (D) EIF4EBP1. Arrows indicate EIF4EBP1 ${ }^{+}$cells. Average with s.e.m. $P$-values by two-sided independent samples $t$-test assuming unequal variances. All stainings were repeated on at least three biological replicates $(n)$. Scale bars: 2 mm in $\mathrm{A} ; 2 \mathrm{~mm}$ (brain), $500 \mu \mathrm{m}$ (cerebellum) and $100 \mu \mathrm{m}$ (EGL and cortex) in B-D.

consistent with recent literature demonstrating that ATR inhibition does not damage non-proliferating, differentiated neurons (Kemp and Sancar, 2016).

\section{DISCUSSION}

Our data show that ATR is required to mitigate endogenous DNA damage during postnatal CGNP proliferation in order to maintain genomic stability. A prior study that deleted Atr using Nes-Cre, which is expressed by E9.5 with an effect by E10.5, found that embryonic CGNP precursors exit the cell cycle prematurely, blocking cerebellar development before postnatal CGNP proliferation (Lee et al., 2012). By contrast, in $\mathrm{Atr}^{G-c r e}$ and $\mathrm{Atr}^{\mathrm{M}-}$ cre mice, where Cre expression does not occur until E11.5 and E12.5 (Andrae et al., 2001; Machold and Fishell, 2005), respectively, CGNPs proliferated normally in the postnatal period and the effect of Atr deletion was not observed until P0 and P3, respectively. 
Postnatal proliferation in Atr-deleted CGNPs produced widespread DNA damage that activated ATM and p53 and induced apoptosis, resulting in cerebellar hypoplasia.

Blocking apoptosis by co-deletion of Bax and Bak or p53 did not fully rescue cerebellar hypoplasia. Cerebellar growth failure persisted despite attenuation of apoptosis due to inappropriate cell cycle exit and differentiation in Atr;Bax;Bak ${ }^{M-c r e}$ CGNPs or caspase-independent cell death in Atr;p53 $3^{\text {M-cre }}$ CGNPs. Karyotype analysis showed that Atr-deleted CGNPs developed extensive chromosomal abnormalities, and RNA-Seq analysis revealed the p53 pathway to be the predominant driver of the transcriptomic response to ATR-deficient proliferation.

Atr deletion in medulloblastoma-prone mice (SmoM2;Atr ${ }^{G-c r e}$ ) completely abrogated tumor formation, suggesting a therapeutic potential for ATR inhibition as a novel treatment for medulloblastoma. The anti-tumor effect of targeting ATR might be magnified in our model, as early deletion of Atr by $h G F A P-C r e$ depletes the pool of CGNPs from which tumors may originate. However, CGNPs were present in P0 Atr ${ }^{G-c r e}$ mice (Fig. 1A), and SmoM2 expression consistently failed to induce tumor growth from these cells in SmoM2;Atr ${ }^{\text {G-cre }}$ animals. Future work will assess the therapeutic potential of the ATR inhibitor pVE-822 in mice with established medulloblastoma. Indeed, we have already shown here that acute in vivo ATR inhibition by $\mathrm{pVE}-822$ in WT mice reproduces the effects of conditional Atr deletion. Taken together, these results define a crucial role for ATR in maintaining genomic integrity in rapidly proliferating neural progenitors and in medulloblastoma cells.

The finding that $5-9 \%$ of Atr-deleted CGNPs were $\mathrm{cC}^{+}$ demonstrates a strong induction of apoptosis. Unlike CGNP death after radiation, the death of these cells is not expected to be a synchronous process. Moreover, dying cells are $\mathrm{cC}^{+}$for only a limited time because they are rapidly cleared by phagocytosis and cC3 is known to have a short half-life (Elliott and Ravichandran, 2010; Walsh et al., 2011). Detecting 5-9\% of cells expressing cC3 thus indicates a high rate of cell death.

Apoptosis in Atr-deleted CGNPs, in contrast to irradiated Atrintact CGNPs (Chong et al., 2000; Williams et al., 2015), was not prevented by co-deletion of Bax, but was prevented by co-deletion of both Bax and Bak. In other cell types, BAK-driven apoptosis can be activated by genotoxic stress during mitosis (Chu et al., 2012; Flores et al., 2012). Atr-deleted CGNPs with DNA damage were markedly more prone to be mitotic than irradiated CGNPs in Atrintact mice. The mitosis of Atr-deleted CGNPs with DNA damage might activate BAK-driven apoptosis that is not activated by radiation in WT mice.

The dependence of CGNPs on ATR to prevent DNA damage suggests that these cells are particularly prone to endogenous replication stress. ATR is activated in response to stalled DNA replication forks (Nam and Cortez, 2011), which can be caused by exogenous (Harley et al., 2016; Harper et al., 2010) or endogenous (Willis et al., 2013) factors. We have previously shown that normal CGNP proliferation produces endogenous DNA damage, detectable as small $\gamma \mathrm{H} 2 \mathrm{~A} . \mathrm{X}^{+}$foci (Williams et al., 2015). We now show that ATR mitigates endogenous, proliferation-associated DNA damage in CGNPs in order to maintain replication fidelity.

To account for the phenotype of Atr deletion we propose a model in which: (1) rapid proliferation promotes replication fork stalling from endogenous sources (Mirkin and Mirkin, 2007); (2) absence of ATR prolongs and collapses forks with consequent formation of DSBs (Couch et al., 2013); (3) ATM is activated and recruited to sites of DSBs (Shiloh, 2003); and (4) p-ATM activates p53, which ultimately induces apoptosis (Shiloh and Ziv, 2013) through BAX and BAK, and cell cycle arrest through p21 (Fig. 7). Because DNA damage and apoptosis occur throughout the entire CGNP population, cerebellar growth is effectively halted.

Apoptosis functions to cull CGNPs that acquire chromosomal abnormalities; thus, disabling apoptosis in ATR-deficient mice increases chromosomal fragmentation. p21 upregulation in Atr;Bax; $B a k^{M-c r e}$ CGNPs demonstrates that $\mathrm{p} 53$-induced cell cycle exit acts as an additional barrier to the propagation of genomic abnormalities in apoptosis-incompetent, Atr-deleted CGNPs. Genomic stability in CGNPs may be particularly important for preventing their rapid postnatal proliferation from degenerating into tumorigenesis. Medulloblastoma, the most common malignant pediatric brain tumor, arises from hindbrain neural progenitors including CGNPs (Yang et al., 2008). Chromosome damage has been observed in medulloblastoma, frequently in association with p53 mutation (Rausch et al., 2012). Importantly, despite severe chromosomal abnormalities in Atr;Bax;Bak $k^{M-c r e}$ and Atr;p53 $3^{M-c r e}$ mice, we never observed tumor formation. Indeed, we found that medulloblastoma tumorigenesis, like cerebellar development, requires ATR.

Since the physiological requirement for ATR in neural progenitors is recapitulated in the pathological growth of medulloblastoma, ATR may be a promising target for novel antitumor therapy. pVE-822 effectively crosses the blood-brain barrier in bioactive concentrations and induces DNA damage in proliferating cells. By damaging DNA in proliferating cells, pVE822 might have a similar toxicity to conventional chemotherapeutic agents such as etoposide. An advantage of ATR inhibition over standard chemotherapy, however, is suggested by our data showing that Atr deletion kills cells even in the absence of p53. Whether the activity of pVE-822 against proliferating, non-tumor cells will limit its therapeutic potential remains to be tested.

\section{MATERIALS AND METHODS}

\section{Mice}

We generated $A t r^{M-c r e}$ and $A t r^{G-c r e}$ mice by crossing Atr loxP/loxP (Brown and Baltimore, 2003) mice with the Math1-Cre (Jackson Labs, stock \#011104) (Matei et al., 2005) and hGFAP-Cre (Jackson Labs, stock \#012886) (Zhuo et al., 2001) mouse lines, respectively. BaxloxP/loxP $; B a k^{-1-}$ mice (Takeuchi et al., 2005) were obtained from Jackson Labs (stock \#006329). To generate mice with co-deletion of Atr/Bax/Bak, Atr/Bax, Atr/Bak and Bax/Bak, we crossed $A t r^{M-c r e}$ and $B a x^{l o x P / l o x P} ; B a k^{-/-}$animals, intercrossed the progeny, and selected mice of Atr;Bax;Bak ${ }^{M-c r e}, A t r ; B a x^{M-c r e}, A t r ; B a k^{M-c r e}$ and Bax; $B a k^{M-c r e}$ genotypes. We generated Atr;p53 $3^{M-c r e}$ mice by crossing Atr ${ }^{M-c r e}$ with $p 53^{\text {loxP/loxP }}$ mice (Jonkers et al., 2001) provided by the NCI (strain $\# 01 X C 2)$. Medulloblastoma-prone animals with and without Atr deletion were born from the cross between $h$ GFAP-Cre;AtrloxP/+ and SmoM2 $2^{\text {loxP/loxP }} ;$ AtrloxP/loxP , in which tumorigenesis is induced by deletion of a loxP-flanked Stop cassette between the Smo promoter and coding region (Schuller et al., 2008). All mice were of species Mus musculus and crossed into the $\mathrm{C} 57 \mathrm{BL} / 6$ background through at least five generations. We used equal numbers of male and female mice, as we did not observe any differences based on sex. Numbers $(n)$ in figures indicate biological replicates, which were determined so as to measure a $25 \%$ difference in means with power $=80 \%$ and $\alpha=0.05$. Animal use was in keeping with the policies of the University of North Carolina at Chapel Hill Institutional Animal Use and Care Committee. P3 mice of the indicated genotypes received 2 Gy whole-body X-ray irradiation and were sacrificed $2 \mathrm{~h}$ later.

\section{Immunostaining of cerebellar sections}

Mouse brains were processed and immunostained as previously described (Gershon et al., 2013). Primary antibodies are listed in the supplementary Materials and Methods. Secondary antibodies of the indicated fluorophores were used at 1:2000 for IF. Cell death was detected by TUNEL assay [ThermoFisher Scientific (TFS) \#C10617] (Galluzzi et al., 2009). DAPI and 
A

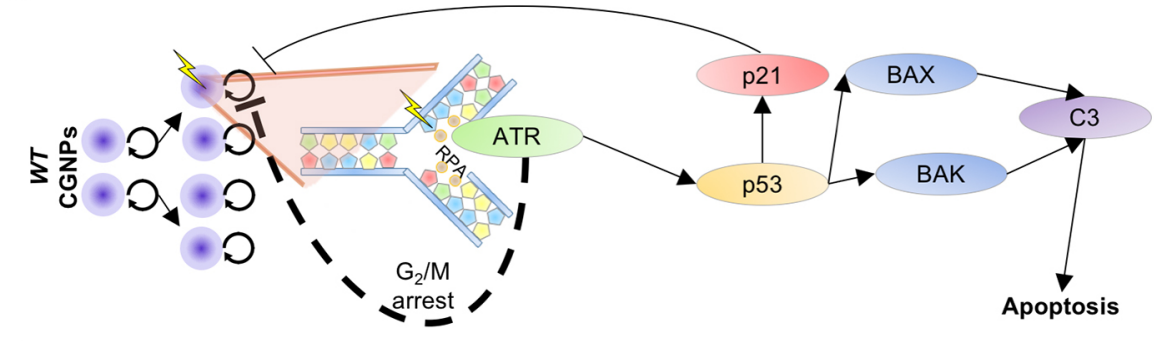

B

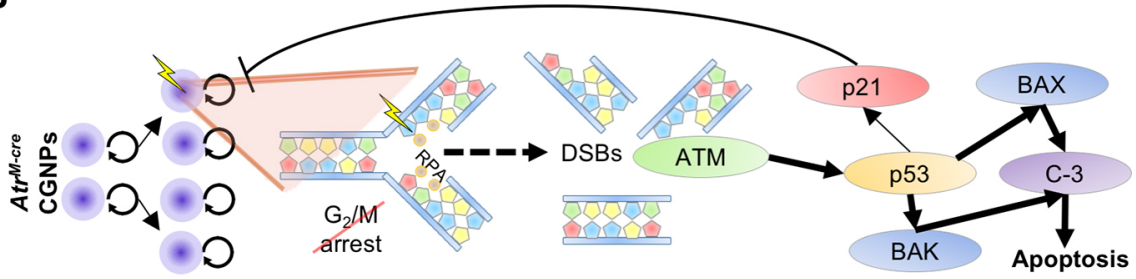

C

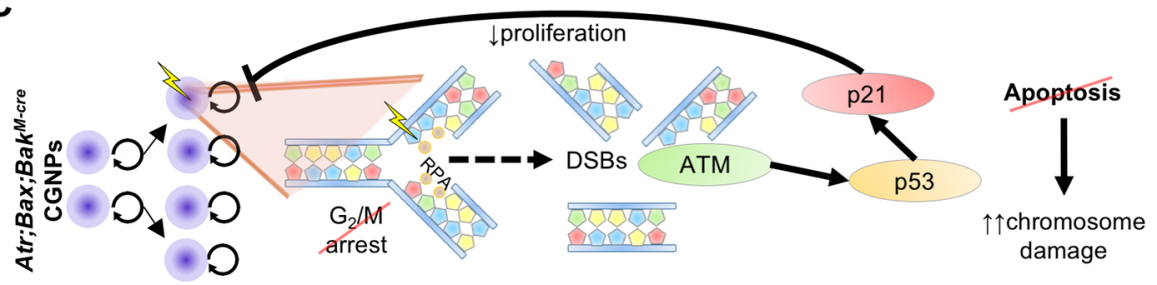

D

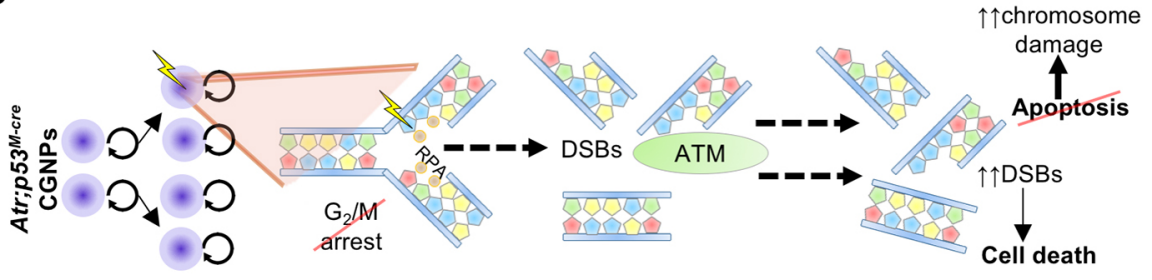

Fig. 7. ATR is required to maintain $G 2 / M$ checkpoint integrity and prevent apoptosis induced by proliferation-associated DNA damage. (A) CGNPs proliferate in the postnatal cerebellum, generating DNA damage (lightning bolt) from endogenous processes, which stalls replication forks. ATR is recruited by replication protein A (RPA), which binds single-stranded DNA at stalled replication forks. Activated ATR initiates checkpoint arrest, promoting DNA repair with minimal engagement of the $p 53$ pathway. (B) ATR loss prolongs replication fork stalling across the CGNP genome, which degenerates into widespread DSBs that are sensed by ATM. Resultant $\mathrm{p} 53$ activation triggers apoptosis through BAX/BAK and caspase 3 (C-3) due to overwhelming DNA damage, with some concurrent activation of p21 and cell cycle arrest. G2/M checkpoint failure from ATR absence allows CGNPs with DNA damage to enter mitosis, incurring chromosomal abnormalities; however, apoptosis removes cells with aberrant chromosomes. (C) Co-deletion of Bax and Bak in ATR-deficient CGNPs prevents apoptosis. Instead, cells with DNA damage and $p 53$ activation are driven to p21-mediated cell cycle exit, attenuating CGNP proliferation over time. However, cells that escape arrest then inappropriately enter mitosis and experience chromosome damage, which accumulates due to apoptotic failure. (D) In Atr; p53 ${ }^{M-c r e}$ CGNPs, DSBs from collapsed replication forks accumulate and propagate due to the inability to undergo both ATR-mediated G2/M arrest and p53-mediated apoptosis and cell cycle arrest. Excessive DNA damage triggers an as yet unidentified form of non-apoptotic cell death.
Hematoxylin were used as nuclear counterstains. BrdU analysis is described in the supplementary Materials and Methods.

\section{Quantification of immunostaining}

Stained slides were digitally imaged and positively stained cells were counted using Aperio Software (Aperio Technologies) for chromogenstained slides or Tissue Studio (Definiens) for fluorescence, as previously described (Williams et al., 2015). The entire EGL region in each section was manually annotated and used for quantifications, which were normalized to the total number of nucleated cells in the designated region. The measurement of cerebellar cellularity is described in the supplementary Materials and Methods.

\section{CGNP isolation}

CGNPs from P3 Atr-deleted mice and controls were isolated as previously described (Lee et al., 2009). Briefly, we separated cerebella from the rest of the brain, removed the meninges, and dissociated the tissue in 20 units $/ \mathrm{ml}$ papain (Worthington Biochemical Corporation \#PDS) at $37^{\circ} \mathrm{C}$ for $15 \mathrm{~min}$. CGNPs were then purified from the dissociated cerebellar tissue by successive rounds of centrifugation, discontinuous density gradient, and mesh filtering.

\section{Flow cytometry}

Flow cytometry on ATR-deficient CGNPs was performed by first fixing and permeabilizing (TFS \#GAS-004) isolated CGNPs suspended in HBSS containing $33 \mathrm{mM}$ glucose, as previously described (Stahl et al., 2008). CGNPs were then stained successively for DNA damage with e660-conjugated anti- $\gamma \mathrm{H} 2 \mathrm{~A} . \mathrm{X}$ Ser139 (eBioscience \#50-9865) at 1:10, for M phase with 488-conjugated anti-pH3 (Cell Signaling Technology \#9708) at 1:25, and for DNA content with FxCycle Violet (TFS \#F10347) at 1:50. Technical controls included no stain, single-stained and fluorescence-minus-one samples. FACS was performed on an LSR Fortessa (BD Biosciences). For all experiments, 10,000-50,000 cells were counted. Analysis of FACS data was performed using FlowJo v10.0.8 (FlowJo).

\section{Cytogenetic analysis}

Cytogenetic analysis was performed on Atr-deleted CGNP metaphase spreads. Freshly isolated CGNPs from P3 animals were treated for $30 \mathrm{~min}$ with $100 \mathrm{nM}$ Colcemid (TFS \#15210-040) in Neurobasal-A Medium (TFS \#10888-022) supplemented with 1× GlutaMAX-I (TFS \#35050), 1× penicillin-streptomycin and $25 \mathrm{mM} \mathrm{KCl}$ at $37^{\circ} \mathrm{C}$ to block cell cycle progression in $\mathrm{M}$ phase. CGNPs were then were resuspended in $75 \mathrm{mM}$ $\mathrm{KCl}$, incubated at $37^{\circ} \mathrm{C}$ for $5 \mathrm{~min}$, and fixed in methanol:acetic acid $(3: 1)$. The fixed cell suspension was dropped onto slides, stained in $0.08 \mu \mathrm{g} / \mathrm{ml}$ DAPI in $2 \times$ SSC for $3 \mathrm{~min}$, and mounted in antifade solution (Vector Laboratories \#H-1200). The stained slides were scanned using a Zeiss Axioplan $2 \mathrm{i}$ epifluorescence microscope equipped with a megapixel CCD camera $\left(\mathrm{CV}-\mathrm{M} 4^{+} \mathrm{CL}\right.$, JAI) controlled by Isis 5.2 imaging software (Metasystems International). Chromatid breaks were counted as singlebreak events, tri-radials and quadri-radials as two-break events each, and other complex chromatid exchanges were converted into the minimum number of breaks required for their theoretical reconstruction. Chromosome aberrations (fragments, rings, dicentric/tricentrics and large marker chromosomes) were recorded and the breaks required for these rearrangements were not added to the frequency of chromatid breaks. 


\section{Mutation analysis}

The frequency of mismatches or of transcript fusions associated with Atr deletion were determined as described in the supplementary Materials and Methods.

\section{Western blot}

P3 WT CGNPs subject to VE-822 or vehicle were analyzed for $\gamma \mathrm{H} 2 \mathrm{~A}$.X and cC3 (with $\beta$-actin loading control) by western blot as described in the supplementary Materials and Methods.

\section{Spectral karyotyping}

Metaphase spreads from the fixed cell suspensions were hybridized with SKY painting probes according to the manufacturer's protocol (Applied Spectral Imaging). SKY images were acquired with an SD300 Spectracube (Applied Spectral Imaging) mounted on a Nikon Eclipse E800 microscope using a custom-designed optical filter (SKY-1) (Chroma Technology). For each sample, a minimum of 20 , but usually 50 , metaphases were captured and fully karyotyped. The breakpoints on the SKY-painted chromosomes were determined by comparison with the corresponding DAPI karyotype and chromosomal abnormalities were described according to ISCN (2013).

\section{RNA-Seq and differential expression analysis}

For RNA-Seq analysis, total RNA was purified using the RNeasy Mini Kit (Qiagen \#74104) from freshly dissected whole P3 cerebella. RNA quality and quantity were assessed by spectrophotometry and capillary gel electrophoresis. We generated stranded mRNA libraries using an Illumina TruSeq Stranded mRNA Library Prep Kit (Illumina \#RS-122-2101). $60 \mathrm{ng} / \mu \mathrm{l}$ poly(A)-selected RNA from each sample was run in two lanes of a HiSeq 2000 sequencing instrument (Illumina) for 100 cycles of multiplexed paired-end reads.

We performed read pseudo-alignment and quantification using Kallisto v0.42.4 [Bray et al. (2015) preprint] with 200 bootstraps/paired-end read, aligning against the mouse $\mathrm{mm} 9$ genomic assembly, GRCm38 transcriptome definition. Downstream analysis was performed using the edgeR v3.12.0 software package (Robinson et al., 2010) in R-Studio v0.99.491 with R v2.11.1 (R Core Team, 2014). To assess differential expression, we used a general linear model in edgeR comparing Atr-deleted with control, setting the minimum counts per million at 2 and requiring greater than half the samples to pass the count threshold for a given transcript. Microarray validation is detailed in the supplementary Materials and Methods.

\section{Pathway analysis}

Biological pathway enrichment was determined by comparing transcriptomic data with Kyoto Encyclopedia of Genes and Genomes v77.1 (Kanehisa and Goto, 2000), WikiPathways (Kutmon et al., 2016) and Gene Ontology v1.2 (Ashburner et al., 2000) databases using EnrichNet v1.1 (Glaab et al., 2012), Panther v10.0 (Mi et al., 2013), and WebGestalt (Wang et al., 2013). Only genes above the differential expression significance threshold of $\log _{2}(\mathrm{FC})>1.5$ and $\mathrm{FDR}<0.01$ were used in pathway analysis.

\section{PVE-822 in vivo administration}

For in vivo studies, WT mice were subject to IP injection with $60 \mathrm{mg} / \mathrm{kg}$ pVE-822 (see supplementary Materials and Methods for formulation) or an equal volume of vehicle every $12 \mathrm{~h}$ for three total injections from P3-5. $12 \mathrm{~h}$ following the final injection, animals were sacrificed and brains were prepared for immunostaining.

\section{Acknowledgements}

We thank the UNC Center for Gastrointestinal Biology and Disease Histology Core for processing tissue sections and Hematoxylin and Eosin staining; the UNC Translational Pathology Laboratory for help in staining and digitizing cerebellar sections; the UNC Flow Cytometry Core Facility for FACS assistance; Jeremy Simon (UNC Neuroscience Center) for transcript fusion analysis on the RNA-Seq data; Matthew Soloway (UNC Lineberger Comprehensive Cancer Center) for uploading RNA-Seq and microarray data to GEO; Jing Gao (UNC Eshelman School of
Pharmacy) for technical assistance with formulating pVE-822; Eric Brown (University of Pennsylvania, Pennsylvania, PA, USA) for AtroxP/loxP mice; David Rowitch (UCSF, San Francisco, CA, USA) and Robert Wechsler-Reya (SanfordBurnham Medical Research Institute, La Jolla, CA, USA) for Math1-Cre mice; and Eva Anton (UNC Neuroscience Center) for hGFAP-Cre mice.

\section{Competing interests}

The authors declare no competing or financial interests

\section{Author contributions}

P.Y.L. and T.R.G. conceived and designed the experiments and wrote the manuscript. G.J.N. and C.S. obtained and analyzed karyotype and SKY data M.S.-P. and A.V.K. conceived the nanoparticle formulation of VE-822 in collaboration with P.Y.L. and T.R.G., and prepared and analyzed pVE-822 with the assistance of D.H. J.S.P. performed mutational analysis and consulted on transcriptomic studies. All other experiments were performed by P.Y.L. and analyzed by P.Y.L. and T.R.G.

\section{Funding}

This work was supported by the National Institute of Neurological Disorders and Stroke [R01NS088219 to T.R.G.]; the National Cancer Institute [F30CA192832 to P.Y.L., P30CA008748 to G.J.N. and C.S., P30CA016086 to J.S.P., and U01CA151806 to D.H., M.S.-P. and A.V.K.]; and Alex's Lemonade Stand Foundation for Childhood Cancer (M.S.-P. and T.R.G.). Deposited in PMC for release after 12 months.

\section{Data availability}

RNA-Seq and microarray data are available at Gene Expression Omnibus under accession number GSE85394 (www.ncbi.nlm.nih.gov/geo/query/acc.cgi? acc=GSE85394)

\section{Supplementary information}

Supplementary information available online at

http://dev.biologists.org/lookup/doi/10.1242/dev.139022.supplemental

\section{References}

Abraham, R. T. (2001). Cell cycle checkpoint signaling through the ATM and ATR kinases. Genes Dev. 15, 2177-2196.

Andrae, J., Bongcam-Rudloff, E., Hansson, I., Lendahl, U., Westermark, B. and Nister, M. (2001). A 1.8kb GFAP-promoter fragment is active in specific regions of the embryonic CNS. Mech. Dev. 107, 181-185.

Ashburner, M., Ball, C. A., Blake, J. A., Botstein, D., Butler, H., Cherry, J. M., Davis, A. P., Dolinski, K., Dwight, S. S., Eppig, J. T. et al. (2000). Gene ontology: tool for the unification of biology. The Gene Ontology Consortium. Nat Genet. 25, 25-29.

Bray, N., Pimentel, H., Melsted, P. and Pachter, L. (2015). Near-optimal RNA-Seq quantification. arXiv: 1505.02710 [q-bio.QM]

Brown, E. J. and Baltimore, D. (2000). ATR disruption leads to chromosomal fragmentation and early embryonic lethality. Genes Dev. 14, 397-402.

Brown, E. J. and Baltimore, D. (2003). Essential and dispensable roles of ATR in cell cycle arrest and genome maintenance. Genes Dev. 17, 615-628

Cai, Z., Jitkaew, S., Zhao, J., Chiang, H.-C., Choksi, S., Liu, J., Ward, Y., Wu, L.G. and Liu, Z.-G. (2014). Plasma membrane translocation of trimerized MLKL protein is required for TNF-induced necroptosis. Nat. Cell Biol. 16, 55-65.

Chandris, P., Giannouli, C. C., Panayotou, G. and Kletsas, D. (2010). Compromise in mRNA processing machinery in senescent human fibroblasts: implications for a novel potential role of phospho-ATR (ser428). Biogerontology 11, 421-436.

Charrier, J.-D., Durrant, S. J., Golec, J. M. C., Kay, D. P., Knegtel, R. M. MacCormick, S., Mortimore, M., O’Donnell, M. E., Pinder, J. L., Reaper, P. M. et al. (2011). Discovery of potent and selective inhibitors of ataxia telangiectasia mutated and Rad3 related (ATR) protein kinase as potential anticancer agents J. Med. Chem. 54, 2320-2330.

Chong, M. J., Murray, M. R., Gosink, E. C., Russell, H. R. C., Srinivasan, A. Kapsetaki, M., Korsmeyer, S. J. and McKinnon, P. J. (2000). Atm and Bax cooperate in ionizing radiation-induced apoptosis in the central nervous system. Proc. Natl. Acad. Sci. USA 97, 889-894.

Chu, R., Terrano, D. T. and Chambers, T. C. (2012). Cdk1/cyclin B plays a key role in mitotic arrest-induced apoptosis by phosphorylation of $\mathrm{Mcl}-1$, promoting its degradation and freeing Bak from sequestration. Biochem. Pharmacol. 83 199-206.

Cimprich, K. A. and Cortez, D. (2008). ATR: an essential regulator of genome integrity. Nat. Rev. Mol. Cell Biol. 9, 616-627.

Couch, F. B., Bansbach, C. E., Driscoll, R., Luzwick, J. W., Glick, G. G., Betous, R., Carroll, C. M., Jung, S. Y., Qin, J., Cimprich, K. A. et al. (2013). ATR phosphorylates SMARCAL1 to prevent replication fork collapse. Genes Dev. 27 
Dobin, A., Davis, C. A., Schlesinger, F., Drenkow, J., Zaleski, C., Jha, S., Batut P., Chaisson, M. and Gingeras, T. R. (2013). STAR: ultrafast universal RNA-seq aligner. Bioinformatics 29, 15-21.

Elliott, M. R. and Ravichandran, K. S. (2010). Clearance of apoptotic cells: implications in health and disease. J. Cell Biol. 189, 1059-1070.

Fingar, D. C., Salama, S., Tsou, C., Harlow, E. and Blenis, J. (2002). Mammalian cell size is controlled by mTOR and its downstream targets S6K1 and 4EBP1/ elF4E. Genes Dev. 16, 1472-1487.

Flores, M. L., Castilla, C., Avila, R., Ruiz-Borrego, M., Saez, C. and Japon, M. A (2012). Paclitaxel sensitivity of breast cancer cells requires efficient mitotic arrest and disruption of $\mathrm{Bcl}-\mathrm{xL} / \mathrm{Bak}$ interaction. Breast Cancer Res. Treat. 133, 917-928

Fokas, E., Prevo, R., Pollard, J. R., Reaper, P. M., Charlton, P. A., Cornelissen, B., Vallis, K. A., Hammond, E. M., Olcina, M. M., Gillies McKenna, W. et al. (2012). Targeting ATR in vivo using the novel inhibitor VE-822 results in selective sensitization of pancreatic tumors to radiation. Cell Death Dis. 3, e441.

Frappart, P.-O., Lee, Y., Lamont, J. and McKinnon, P. J. (2007). BRCA2 is required for neurogenesis and suppression of medulloblastoma. EMBO J. 26 2732-2742

Frappart, P.-O., Lee, Y., Russell, H. R., Chalhoub, N., Wang, Y.-D., Orii, K. E., Zhao, J., Kondo, N., Baker, S. J. and McKinnon, P. J. (2009). Recurrent genomic alterations characterize medulloblastoma arising from DNA doublestrand break repair deficiency. Proc. Natl. Acad. Sci. USA 106, 1880-1885.

Galluzzi, L., Aaronson, S. A., Abrams, J., Alnemri, E. S., Andrews, D. W., Baehrecke, E. H., Bazan, N. G., Blagosklonny, M. V., Blomgren, K., Borner, C. et al. (2009). Guidelines for the use and interpretation of assays for monitoring cell death in higher eukaryotes. Cell Death Differ. 16, 1093-1107.

Garcia, I., Crowther, A. J., Gama, V., Ryan Miller, C., Deshmukh, M. and Gershon, T. R. (2013). Bax deficiency prolongs cerebellar neurogenesis accelerates medulloblastoma formation and paradoxically increases both malignancy and differentiation. Oncogene 32, 2304-2314.

Gershon, T. R., Crowther, A. J., Tikunov, A., Garcia, I., Annis, R., Yuan, H., Miller, C. R., Macdonald, J., Olson, J. and Deshmukh, M. (2013). Hexokinase2-mediated aerobic glycolysis is integral to cerebellar neurogenesis and pathogenesis of medulloblastoma. Cancer Metab. 1, 2.

Glaab, E., Baudot, A., Krasnogor, N., Schneider, R. and Valencia, A. (2012) EnrichNet: network-based gene set enrichment analysis. Bioinformatics 28 i451-i457.

Harley, M. E., Murina, O., Leitch, A., Higgs, M. R., Bicknell, L. S., Yigit, G. Blackford, A. N., Zlatanou, A., Mackenzie, K. J., Reddy, K. et al. (2016). TRAIP promotes DNA damage response during genome replication and is mutated in primordial dwarfism. Nat. Genet. 48, 36-43.

Harper, J. V., Anderson, J. A. and O'Neill, P. (2010). Radiation induced DNA DSBs: contribution from stalled replication forks? DNA Repair 9, 907-913.

Hatten, M. E. and Heintz, N. (1995). Mechanisms of neural patterning and specification in the development cerebellum. Annu. Rev. Neurosci. 18, 385-408.

Hatten, M. E. and Roussel, M. F. (2011). Development and cancer of the cerebellum. Trends Neurosci. 34, 134-142.

Herzog, K.-H., Chong, M. J., Kapsetaki, M., Morgan, J. I. and McKinnon, P. J. (1998). Requirement for Atm in ionizing radiation-induced cell death in the developing central nervous system. Science 280, 1089-1091.

Holcomb, V. B., Vogel, H., Marple, T., Kornegay, R. W. and Hasty, P. (2006). Ku80 and p53 suppress medulloblastoma that arise independent of Rag-1-induced DSBs. Oncogene 25, 7159-7165.

ISCN (2013). An Internationl System for Human Cytogenetic Nomenclature. Basel, Switzerland: S. Karger.

Jonkers, J., Meuwissen, R., van der Gulden, H., Peterse, H., van der Valk, M. and Berns, A. (2001). Synergistic tumor suppressor activity of BRCA2 and p53 in a conditional mouse model for breast cancer. Nat. Genet. 29, 418-425.

Kanehisa, M. and Goto, S. (2000). KEGG: kyoto encyclopedia of genes and genomes. Nucleic Acids Res. 28, 27-30.

Kemp, M. G. and Sancar, A. (2016). ATR kinase inhibition protects non-cycling cells from the lethal effects of DNA damage and transcription stress. J. Biol. Chem. 291, 9330-9342.

Kutmon, M., Riutta, A., Nunes, N., Hanspers, K., Willighagen, E. L., Bohler, A., Melius, J., Waagmeester, A., Sinha, S. R., Miller, R. et al. (2016). WikiPathways: capturing the full diversity of pathway knowledge. Nucleic Acids Res. 44 D488-D494.

Langmead, B., Trapnell, C., Pop, M. and Salzberg, S. L. (2009). Ultrafast and memory-efficient alignment of short DNA sequences to the human genome. Genome Biol. 10, R25.

Lee, Y. and McKinnon, P. J. (2002). DNA ligase IV suppresses medulloblastoma formation. Cancer Res. 62, 6395-6399.

Lee, Y. and McKinnon, P. J. (2007). Responding to DNA double strand breaks in the nervous system. Neuroscience 145, 1365-1374.

Lee, H. Y., Greene, L. A., Mason, C. A. and Manzini, M. C. (2009). Isolation and culture of post-natal mouse cerebellar granule neuron progenitor cells and neurons. J. Vis. Exp. 23, pii: 990.
Lee, Y., Shull, E. R. P., Frappart, P.-O., Katyal, S., Enriquez-Rios, V., Zhao, J. Russell, H. R., Brown, E. J. and McKinnon, P. J. (2012). ATR maintains select progenitors during nervous system development. EMBO J. 31, 1177-1189.

Lovejoy, C. A. and Cortez, D. (2009). Common mechanisms of PIKK regulation. DNA Repair 8, 1004-1008.

Luxenhofer, R., Schulz, A., Roques, C., Li, S., Bronich, T. K., Batrakova, E. V., Jordan, R. and Kabanov, A. V. (2010). Doubly amphiphilic poly(2-oxazoline)s as high-capacity delivery systems for hydrophobic drugs. Biomaterials $\mathbf{3 1}$, 4972-4979.

Machold, R. and Fishell, G. (2005). Math1 is expressed in temporally discrete pools of cerebellar rhombic-lip neural progenitors. Neuron 48, 17-24.

Matei, V., Pauley, S., Kaing, S., Rowitch, D., Beisel, K. W., Morris, K., Feng, F., Jones, K., Lee, J. and Fritzsch, B. (2005). Smaller inner ear sensory epithelia in Neurog1 null mice are related to earlier hair cell cycle exit. Dev. Dyn. 234 633-650.

McMahon, J. J., Shi, L. and Silver, D. L. (2014). Generation of a Magoh conditional allele in mice. Genesis 52, 752-758.

Mi, H., Muruganujan, A., Casagrande, J. T. and Thomas, P. D. (2013). Largescale gene function analysis with the PANTHER classification system. Nat. Protoc. 8, 1551-1566.

Mirkin, E. V. and Mirkin, S. M. (2007). Replication fork stalling at natura impediments. Microbiol. Mol. Biol. Rev. 71, 13-35.

Nam, E. A. and Cortez, D. (2011). ATR signalling: more than meeting at the fork. Biochem. J. 436, 527-536.

Nicorici, D., Satalan, M., Edgren, H., Kangaspeska, S., Murumagi, A. Kallioniemi, O., Virtanen, S. and Kilkku, O. (2014). FusionCatcher - a tool for finding somatic fusion genes in paired-end RNA-sequencing data. bioRxiv http://dx.doi.org/10.1101/011650.

O'Driscoll, M., Ruiz-Perez, V. L., Woods, C. G., Jeggo, P. A. and Goodship, J. A. (2003). A splicing mutation affecting expression of ataxia-telangiectasia and Rad3-related protein (ATR) results in Seckel syndrome. Nat. Genet. 33 497-501.

Orii, K. E., Lee, Y., Kondo, N. and McKinnon, P. J. (2006). Selective utilization of nonhomologous end-joining and homologous recombination DNA repair pathways during nervous system development. Proc. Natl. Acad. Sci. USA 103 10017-10022.

R Core Team. (2014). R: A Language and Environment for Statistical Computing. $\mathrm{R}$ Foundation for Statistical Computing, Vienna, Austria. https://www.r-project.org.

Rausch, T., Jones, D. T. W., Zapatka, M., Stutz, A. M., Zichner, T., Weischenfeldt J., Jager, N., Remke, M., Shih, D., Northcott, P. A. et al. (2012). Genome sequencing of pediatric medulloblastoma links catastrophic DNA rearrangements with TP53 mutations. Cell 148, 59-71.

Robinson, M. D., McCarthy, D. J. and Smyth, G. K. (2010). edgeR: a Bioconductor package for differential expression analysis of digital gene expression data. Bioinformatics 26, 139-140.

Rosin, N., Elcioglu, N. H., Beleggia, F., Isguven, P., Altmuller, J., Thiele, H., Steindl, K., Joset, P., Rauch, A., Nurnberg, P. et al. (2015). Mutations in XRCC4 cause primary microcephaly, short stature and increased genomic instability. Hum. Mol. Genet. 24, 3708-3717.

Schuller, U., Heine, V. M., Mao, J., Kho, A. T., Dillon, A. K., Han, Y.-G., Huillard, E., Sun, T., Ligon, A. H., Qian, Y. et al. (2008). Acquisition of granule neuron precursor identity is a critical determinant of progenitor cell competence to form Shh-induced medulloblastoma. Cancer Cell 14, 123-134.

Shiloh, Y. (2003). ATM and related protein kinases: safeguarding genome integrity Nat. Rev. Cancer 3, 155-168.

Shiloh, Y. and Ziv, Y. (2013). The ATM protein kinase: regulating the cellular response to genotoxic stress, and more. Nat. Rev. Mol. Cell Biol. 14, 197-210.

Smith, J., Tho, L. M., Xu, N. and Gillespie, D. A. (2010). The ATM-Chk2 and ATRChk1 pathways in DNA damage signaling and cancer. Adv. Cancer Res. 108 73-112.

Stahl, M., Uemura, K., Ge, C., Shi, S., Tashima, Y. and Stanley, P. (2008). Roles of Pofut1 and O-fucose in mammalian Notch signaling. J. Biol. Chem. 283, 13638-13651.

Takeuchi, O., Fisher, J., Suh, H., Harada, H., Malynn, B. A. and Korsmeyer, S. J. (2005). Essential role of BAX,BAK in B cell homeostasis and prevention of autoimmune disease. Proc. Natl. Acad. Sci. USA 102, 11272-11277.

Tong, W.-M., Ohgaki, H., Huang, H., Granier, C., Kleihues, P. and Wang, Z.-Q. (2003). Null mutation of DNA strand break-binding molecule poly(ADP-ribose) polymerase causes medulloblastomas in p53(-/-) mice. Am. J. Pathol. 162 343-352.

Vakifahmetoglu, H., Olsson, M., Tamm, C., Heidari, N., Orrenius, S. and Zhivotovsky, B. (2008). DNA damage induces two distinct modes of cell death in ovarian carcinomas. Cell Death Differ. 15, 555-566.

Walsh, J. G., Logue, S. E., Luthi, A. U. and Martin, S. J. (2011). Caspase-1 promiscuity is counterbalanced by rapid inactivation of processed enzyme. J. Biol. Chem. 286, 32513-32524

Wang, K., Singh, D., Zeng, Z., Coleman, S. J., Huang, Y., Savich, G. L., He, X., Mieczkowski, P., Grimm, S. A., Perou, C. M. et al. (2010). MapSplice: accurate mapping of RNA-seq reads for splice junction discovery. Nucleic Acids Res. 38 e178. 
Wang, J., Duncan, D., Shi, Z. and Zhang, B. (2013). WEB-based GEne SeT AnaLysis Toolkit (WebGestalt): update 2013. Nucleic Acids Res. 41, W77-W83.

Williams, S. E., Garcia, I., Crowther, A. J., Li, S., Stewart, A., Liu, H., Lough, K. J., O'Neill, S., Veleta, K., Oyarzabal, E. A. et al. (2015). Aspm sustains postnatal cerebellar neurogenesis and medulloblastoma growth in mice. Development 142, 3921-3932.

Willis, J., Patel, Y., Lentz, B. L. and Yan, S. (2013). APE2 is required for ATR-Chk1 checkpoint activation in response to oxidative stress. Proc. Natl. Acad. Sci. USA 110, 10592-10597.
Yang, Z.-J., Ellis, T., Markant, S. L., Read, T.-A., Kessler, J. D., Bourboulas, M., Schuller, U., Machold, R., Fishell, G., Rowitch, D. H. et al. (2008). Medulloblastoma can be initiated by deletion of Patched in lineage-restricted progenitors or stem cells. Cancer Cell 14, 135-145.

Zhuo, L., Theis, M., Alvarez-Maya, I., Brenner, M., Willecke, K. and Messing, A. (2001). hGFAP-cre transgenic mice for manipulation of glial and neuronal function in vivo. Genesis 31, 85-94.

Zuckerman, V., Wolyniec, K., Sionov, R. V., Haupt, S. and Haupt, Y. (2009).

Tumour suppression by p53: the importance of apoptosis and cellular senescence. J. Pathol. 219, 3-15. 